\title{
Perturbation of Symmetric Markov Processes
}

\author{
Z.-Q. Chen* P. J. Fitzsimmons $₫$ K. Kuwae ${ }^{\ddagger}$ and T.-S. Zhang ${ }^{\S}$
}

(Version of February 21, 2007)

In Memory of Professor Martin L. Silverstein

\begin{abstract}
We present a path-space integral representation of the semigroup associated with the quadratic form obtained by a lower-order perturbation of the $L^{2}$-infinitesimal generator $\mathcal{L}$ of a general symmetric Markov process. An illuminating concrete example for $\mathcal{L}$ is $\Delta_{D}-(-\Delta)_{D}^{s}$, where $D$ is a bounded Euclidean domain in $\left.\mathbb{R}^{d}, s \in\right] 0,1\left[, \Delta_{D}\right.$ is the Laplace operator in $D$ with zero Dirichlet boundary condition and $-(-\Delta)_{D}^{s}$ is the fractional Laplacian in $D$ with zero exterior condition. The strong Markov process corresponding to $\mathcal{L}$ is a Lévy process that is the sum of Brownian motion in $\mathbb{R}^{d}$ and an independent symmetric $(2 s)$-stable process in $\mathbb{R}^{d}$ killed upon exiting the domain $D$. This probabilistic representation is a combination of Feynman-Kac and Girsanov formulas. Crucial to the development is the use of an extension of Nakao's stochastic integral for zero-energy additive functionals and the associated Itô formula, both of which were recently developed in [3].
\end{abstract}

AMS 2000 Mathematics Subject Classification: Primary 31C25; Secondary 60J57, 60J55, $60 \mathrm{H} 05$.

Keywords and phrases: Perturbation, symmetric Markov process, time reversal, Girsanov transform, Feynman-Kac transform, stochastic integral for Dirichlet processes, martingale, Revuz measure, dual predictable projection.

\footnotetext{
${ }^{*}$ The research of this author is supported in part by NSF Grant DMS-0600206.

${ }^{\dagger}$ The research of this author is supported by a foundation based on the academic cooperation between Yokohama City University and UCSD

${ }^{\ddagger}$ The research of this author is supported by a foundation based on the academic cooperation between Yokohama City University and UCSD, and partially supported by a Grant-in-Aid for Scientific Research (C) No. 16540201 from Japan Society for the Promotion of Science.

$\S$ The research of this author is supported by the British EPSRC.
} 


\section{Introduction}

Let $A(x):=\left(a_{i j}(x)\right)_{1 \leq i, j \leq d}$ be a symmetric matrix-valued function on $\mathbb{R}^{n}$ that is uniformly elliptic and bounded. It is well-known that there is a conservative symmetric diffusion $\left\{\Omega, X, \mathbf{P}_{x}, x \in \mathbb{R}^{d}\right\}$ on $\mathbb{R}^{d}$ with infinitesimal generator $\mathcal{L}:=\frac{1}{2} \sum_{i, j=1}^{d} \frac{\partial}{\partial x_{i}}\left(a_{i j}(x) \frac{\partial}{\partial x_{j}}\right)$. Moreover $X$ has the following Fukushima decomposition (see [11])

$$
X_{t}=X_{0}+M_{t}+N_{t}, \quad t \geq 0
$$

where $M=\left(M^{1}, \cdots, M^{d}\right)$ is a square-integrable martingale additive functional of $X$ with quadratic covariation $\left\langle M^{i}, M^{j}\right\rangle_{t}=\int_{0}^{t} a_{i j}\left(X_{s}\right) d s$ and $N=\left(N^{1}, \cdots, N^{d}\right)$ is a continuous additive functional of $X$ locally of zero energy. Let $b$ and $\widehat{b}$ be two $\mathbb{R}^{d}$-valued functions on $\mathbb{R}^{d}$, and $c$ a measurable function on $\mathbb{R}^{d}$ such that $|b|+|\widehat{b}| \in L^{p_{1}}\left(\mathbb{R}^{d}\right)$ for some $p_{1}>d$ (resp. $p_{1} \geq 2$ ) and $c \in L^{p_{1}}\left(\mathbb{R}^{d}\right)$ for some $p_{2}>d / 2\left(\right.$ resp. $\left.p_{2} \geq 1\right)$ in case $d \geq 2$ (resp. $d=1$ ). In [16], Lunt, Lyons and Zhang showed that the semigroup $\left\{T_{t}, t \geq 0\right\}$ generated by the following operator

$$
\widetilde{\mathcal{L}} \varphi(x)=\frac{1}{2} \sum_{i, j=1}^{d} \frac{\partial}{\partial x_{i}}\left(a_{i j}(x) \frac{\partial \varphi(x)}{\partial x_{j}}\right)+b(x) \cdot \nabla \varphi(x)-\operatorname{div}(\widehat{b}(x) \varphi(x))+c(x) \varphi(x)
$$

is given by

$$
T_{t} f(x)=\mathbf{E}_{x}\left[Z_{t} f\left(X_{t}\right)\right]
$$

where

$$
\begin{aligned}
Z_{t}=\exp ( & \int_{0}^{t}\left(A^{-1} b\right)\left(X_{s}\right) \cdot d M_{s}+\left(\int_{0}^{t}\left(A^{-1} \widehat{b}\right)\left(X_{s}\right) \cdot d M_{s}\right) \circ r_{t} \\
& \left.-\frac{1}{2} \int_{0}^{t}\left((b-\widehat{b}) \cdot A^{-1}(b-\widehat{b})\right)\left(X_{s}\right) d s+\int_{0}^{t} c\left(X_{s}\right) d s\right) .
\end{aligned}
$$

Here $r_{t}$ is the time-reversal operator on $\Omega$ from time $t>0$; that is, given a path $\omega \in \Omega$,

$$
r_{t}(\omega)(s):= \begin{cases}\omega(t-s), & \text { if } 0 \leq s \leq t, \\ \omega(0), & \text { if } s \geq t\end{cases}
$$

Recently, Fitzsimmons and Kuwae [10] extended the above result from symmetric diffusions $X$ on $\mathbb{R}^{d}$ associated with bounded uniformly elliptic divergence-form operators to general symmetric diffusions with no killing inside the state space. The purpose of this paper is to establish similar results for general symmetric Markov processes which may have discontinuous sample paths and killing inside the state space. An illuminating concrete example to keep in mind while reading this paper is that of $X$ a discontinuous symmetric Lévy process killed upon exiting a domain, such as the sum of a Brownian motion on $\mathbb{R}^{d}$ and an independent symmetric $\alpha$-stable process on $\mathbb{R}^{d}$ that is killed upon leaving an open ball. When $X$ is a discontinuous symmetric Markov process, its martingale additive functionals may be discontinuous. These discontinuities pose many challenges when studying transformations of $X$ of a form analogous to that found in (1.3). One 
of the challenges is to define stochastic integral for zero-energy additive functionals of $X$ and to establish the associated Itô formula. Nakao [19] has defined such a stochastic integral for a class of integrands that is too restrictive for our investigation. In our recent paper [3], we established the necessary stochastic integration theory for zero-energy additive functionals of $X$ as well as the corresponding Itô formula via a time-reversal technique. The main result of the current paper extends not only the results in [16] and [10] but also the Feynman-Kac transforms by continuous additive functionals of zero energy studied in Chen and Zhang [8] and the pure-jump Girsanov transforms and discontinuous Feynman-Kac transforms in Chen [1] and in Chen and Song [5]-[6]. The following is a more detailed description of this paper.

Throughout this paper, $X=\left(\Omega, \mathcal{F}_{\infty}, \mathcal{F}_{t}, X_{t}, \zeta, \mathbf{P}_{x}, x \in E\right)$ is an $m$-symmetric right Markov process on a Lusin space $E$, where $m$ is a positive $\sigma$-finite measure with full topological support on $E$. A cemetery state $\partial$ is added to $E$ to form $E_{\partial}:=E \cup\{\partial\}$, and $\Omega$ is the totality of rightcontinuous, left-limited ( $r c l l$, for short) sample paths from $\left[0, \infty\left[\right.\right.$ to $E_{\partial}$ that hold the value $\partial$ once attaining it. For any $\omega \in \Omega$, we set $X_{t}(\omega):=\omega(t)$. Let $\zeta(\omega):=\inf \left\{t \geq 0 \mid X_{t}(\omega)=\partial\right\}$ be the life time of $X$. As usual, $\mathcal{F}_{\infty}$ and $\mathcal{F}_{t}$ are the minimal augmented $\sigma$-algebras obtained from $\mathcal{F}_{\infty}^{0}:=\sigma\left\{X_{s} \mid 0 \leq s<\infty\right\}$ and $\mathcal{F}_{t}^{0}:=\sigma\left\{X_{s} \mid 0 \leq s \leq t\right\}$ under $\mathbf{P}_{x}$; see Section 3 below for more details. We sometimes use a filtration denoted by $\left(\mathcal{M}_{t}\right)$ on $(\Omega, \mathcal{M})$ in order to represent several filtrations, for example, $\left(\mathcal{F}_{t}^{0}\right),\left(\mathcal{F}_{t+}^{0}\right)$ on $\left(\Omega, \mathcal{F}_{\infty}^{0}\right),\left(\mathcal{F}_{t}\right)$ on $\left(\Omega, \mathcal{F}_{\infty}\right)$ and others introduced later. We use $\theta_{t}$ to denote the shift operator defined by $\theta_{t}(\omega)(s):=\omega(t+s), t, s \geq 0$. Let $\omega_{\partial}$ be the path starting from $\partial$. Then $\omega_{\partial}(s) \equiv \partial$ for all $s \in\left[0, \infty\left[\right.\right.$. Note that $\theta_{\zeta(\omega)}(\omega)=\omega_{\partial}$ for all $\omega \in \Omega$, $\left\{\omega_{\partial}\right\} \in \mathcal{F}_{0}^{0} \subset \mathcal{F}_{t}^{0}$ for all $t>0$ and $\mathbf{P}_{x}\left(\left\{\omega_{\partial}\right\}\right) \leq \mathbf{P}_{x}\left(X_{0}=\partial\right)=0$ for $x \in E$. For a Borel subset $B$ of $E, \tau_{B}:=\inf \left\{t>0 \mid X_{t} \notin B\right\}$ (the exit time of $B$ ) is an $\left(\mathcal{F}_{t}\right)$-stopping time. If $B$ is closed, then $\tau_{B}$ is an $\left(\mathcal{F}_{t+}^{0}\right)$-stopping time. Also, $\zeta$ is an $\left(\mathcal{F}_{t}^{0}\right)$-stopping time because $\{\zeta \leq t\}=\left\{X_{t}=\partial\right\} \in \mathcal{F}_{t}^{0}$, $t \geq 0$.

The transition semigroup of $X,\left\{P_{t}, t \geq 0\right\}$, is defined by

$$
P_{t} f(x):=\mathbf{E}_{x}\left[f\left(X_{t}\right)\right]=\mathbf{E}_{x}\left[f\left(X_{t}\right): t<\zeta\right], \quad t \geq 0 .
$$

Each $P_{t}$ may be viewed as an operator on $L^{2}(E ; m)$; collectively these operators form a strongly continuous semigroup of self-adjoint contractions. The Dirichlet form associated with $X$ is the bilinear form

$$
\mathcal{E}(u, v):=\lim _{t \downarrow 0} \frac{1}{t}\left(u-P_{t} u, v\right)_{m}
$$

defined on the space

$$
\mathcal{F}:=\left\{u \in L^{2}(E ; m) \mid \sup _{t>0} t^{-1}\left(u-P_{t} u, u\right)_{m}<\infty\right\}
$$

Here we use the notation $(f, g)_{m}:=\int_{E} f(x) g(x) m(d x)$. Since $(\mathcal{E}, \mathcal{F})$ is a quasi-regular Dirichlet form, we know from $[4]$ that $(\mathcal{E}, \mathcal{F})$ is quasi-homeomorphic to a regular Dirichlet form on a locally compact metric space. Thus without loss of generality, we may and do assume that $X$ is an $m$ symmetric Hunt process on a locally compact metric space $E$, whose associated Dirichlet form 
$(\mathcal{E}, \mathcal{F})$ is regular in $L^{2}(E ; m)$ and that $m$ is a positive Radon measure on $E$ with full topological support. For notions such as quasi-continuous, quasi-everywhere (abbreviated as q.e. or $\mathcal{E}$-q.e.), $\mathcal{E}$ nest, martingale additive functional, continuous additive functionals, $\mathcal{F}_{\text {loc }}$, etc. we refer the reader to [11] and [17]. In particular, we recall that an increasing sequence of closed sets $\left\{F_{n}\right\}$ is an $\mathcal{E}$-nest if $\cup_{n=1}^{\infty} \mathcal{F}_{F_{n}}$ is $\mathcal{E}_{1}^{1 / 2}$-dense in $\mathcal{F}$, where $\mathcal{E}_{1}(u, u):=\mathcal{E}(u, u)+(u, u)_{m}$ and $\mathcal{F}_{F_{n}}:=\{u \in \mathcal{F}: u=$ $0 m$-a.e. on $E \backslash F_{n}$ \}. A function $f$ is said to be locally in $\mathcal{F}$ (denoted as $f \in \mathcal{F}_{\text {loc }}$ ) if there is an increasing sequence of finely open Borel sets $\left\{D_{k}, k \geq 1\right\}$ with $\cup_{k=1}^{\infty} D_{k}=E$ q.e. and for every $k \geq 1$, there is $f_{k} \in \mathcal{F}$ such that $f=f_{k} m$-a.e. on $D_{k}$.

The main purpose of this paper is to establish a probabilistic representation (via a combination of Girsanov and Feynman-Kac transformations) of the semigroups of certain lower-order perturbations of the Dirichlet form $\mathcal{E}$. To discuss these perturbations we need to establish some notation.

A positive continuous additive functional (PCAF in abbreviation) of $X$ (call it $A$ ) determines a measure $\mu=\mu_{A}$ on the Borel subsets of $E$ via the formula

$$
\int_{E} f(x) \mu(d x)=\uparrow \lim _{t \rightarrow 0} \frac{1}{t} \mathbf{E}_{m}\left[\int_{0}^{t} f\left(X_{s}\right) d A_{s}\right],
$$

in which $f: E \rightarrow[0, \infty]$ is Borel measurable. The measure $\mu$ is necessarily smooth, in the sense that $\mu$ charges no exceptional set of $X$ and there is an $\mathcal{E}$-nest $\left\{F_{n}\right\}$ of closed subsets of $E$ such that $\mu\left(F_{n}\right)<\infty$ for each $n \in \mathbb{N}$. Conversely, given a smooth measure $\mu$, there is a unique PCAF $A^{\mu}$ such that (1.4) holds with $A=A^{\mu}$. In the sequel we refer to this bijection between smooth measures and PCAFs as the Revuz correspondence, and to $\mu$ as the Revuz measure of $A^{\mu}$. A smooth measure $\nu$ is said to be of the Hardy class if there are constants $\delta>0$ and $\gamma \geq 0$ such that

$$
\int_{E} \tilde{u}^{2} d \nu \leq \delta \cdot \mathcal{E}(u, u)+\gamma \cdot(u, u)_{m} \quad \text { for every } u \in \mathcal{F} .
$$

It is well known that for every $u \in \mathcal{F}, u$ has a quasi-continuous $m$-version $\tilde{u}$. As a rule we take $u$ to be represented by its quasi-continuous version (when such exists), and drop the tilde from the notation. Let $\stackrel{\circ}{\mathcal{M}}$ and $\mathcal{N}$ denote, respectively, the space of MAFs of finite energy and the space of continuous additive functionals of zero energy. For $u \in \mathcal{F}$, the following Fukushima decomposition holds:

$$
u\left(X_{t}\right)-u\left(X_{0}\right)=M_{t}^{u}+N_{t}^{u} \quad \text { for } t \in[0, \infty[,
$$

$\mathbf{P}_{x^{-}}$a.s. for $\mathcal{E}$-q.e. $x \in E$, where $M^{u} \in \stackrel{\circ}{\mathcal{M}}$ and $N^{u} \in \mathcal{N}$.

If $M$ is a locally square-integrable martingale additive functional (MAF) on $\llbracket 0, \zeta \llbracket$ of $X$, then the process $\langle M\rangle$ (the dual predictable projection of $[M]$ ) is a PCAF, and the associated Revuz measure (as in (1.4)) is denoted by $\mu_{\langle M\rangle}$ (see [3]). More generally, if $M^{u}$ is the martingale part in the Fukushima decomposition (1.5) of $u \in \mathcal{F}$, then $\left\langle M^{u}, M\right\rangle$ is a CAF locally of bounded variation, and we have the associated Revuz measure $\mu_{\left\langle M^{u}, M\right\rangle}$, which is locally the difference of smooth (positive) measures.

Now let $M$ and $\widehat{M}$ be two locally square-integrable MAFs on $I(\zeta)$ such that $\mu_{\langle M\rangle}$ and $\mu_{\langle\widehat{M}\rangle}$ are of the Hardy class, and let $A^{\mu}$ be a CAF locally of bounded variation whose Revuz measure $\mu$ has 
total variation $|\mu|$ of the Hardy class. Here $I(\zeta):=\llbracket 0, \zeta \llbracket \cup \llbracket \zeta_{i} \rrbracket$, where $\zeta_{i}$ is the totally inaccessible part of $\zeta$ (see the comment before Definition 2.1). As the main result of this paper (Theorem 3.1), we show that under a suitable condition on the $\delta$ coefficients in the Hardy inequality for $\mu_{\langle M\rangle}$, $\mu_{\langle\widehat{M}\rangle}$, and $|\mu|$, the form perturbation $(\mathcal{Q}, \mathcal{F})$ of $(\mathcal{E}, \mathcal{F})$ defined by

$$
\begin{aligned}
\mathcal{Q}(f, g)=\mathcal{E}(f, g) & -\int_{E} f(x) \mu_{\left\langle M^{g}, \widehat{M}\right\rangle}(d x)-\int_{E} g(x) \mu_{\left\langle M^{f}, M\right\rangle}(d x)-\int_{E} f(x) g(x) \mu(d x) \\
& -\int_{E \times E} f(y) g(x) \varphi(x, y) \psi(y, x) N(x, d y) \mu_{H}(d x) .
\end{aligned}
$$

determines a strongly continuous semigroup $\left\{T_{t}, t \geq 0\right\}$ of operators on $L^{2}(E ; m)$, where $\varphi$ and $\psi$ are Borel functions bounded below away from -1 , coming from the jump parts of $M$ and $\widehat{M}$ respectively, and $\left\{T_{t}, t \geq 0\right\}$ admits the representation

$$
T_{t} f(x):=\mathbf{E}_{x}\left[Z_{t} f\left(X_{t}\right)\right]
$$

where

$$
Z_{t}=\operatorname{Exp}\left(M_{t}+A_{t}^{\mu}+\left\langle M^{c}, \widehat{M}^{c}\right\rangle_{t}\right) \cdot \operatorname{Exp}\left(\widehat{M}_{t}\right) \circ r_{t} \cdot\left(1+\psi\left(X_{t}, X_{t-}\right)\right) \quad \text { for } t<\zeta
$$

Here $r_{t}$ is the time-reversal operator defined on the path space $\Omega$ of $X$ as follows: Given a path $\omega \in\{t<\zeta\}$,

$$
r_{t}(\omega)(s)= \begin{cases}\omega\left((t-s)_{-}\right), & \text {if } 0 \leq s \leq t, \\ \omega(0), & \text { if } s \geq t,\end{cases}
$$

in which, for $r>0, \omega\left(r_{-}\right):=\lim _{s \uparrow r} \omega(s)$. (The restriction of the measure $\mathbf{P}_{m}$ to $\mathcal{F}_{t}$ is invariant under $r_{t}$ on $\Omega \cap\{\zeta>t\}$.) Also in (1.6), the symbol Exp denotes the familiar Doléans-Dade stochastic exponential: if $Y$ is a semimartingale with $Y_{0}=0$, then $Z=\operatorname{Exp}(Y)$ is the unique solution of the SDE

$$
Z_{t}=1+\int_{0}^{t} Z_{s-} d Y_{s}
$$

and is given explicitly by the formula

$$
\operatorname{Exp}\left(Y_{t}\right)=\exp \left(Y_{t}-\frac{1}{2}\left\langle Y^{c}, Y^{c}\right\rangle_{t}\right) \prod_{s \in] 0, t]}\left(1+\Delta Y_{s}\right) e^{-\Delta Y_{s}}
$$

As mentioned previously, this result was first obtained by Lunt, Lyons and Zhang [16] when $X$ is a conservative symmetric diffusion on $\mathbb{R}^{d}$ whose $L^{2}$-infinitesimal generator is an elliptic operator of divergence form, and then by Fitzsimmons and Kuwae [10] in case $X$ is a diffusion process on a Lusin space $E$ with no killing inside $E$. The jumps of $X$, as allowed in the context of the present paper, complicate the study. To deal with these complications we are compelled to develop certain aspects of the stochastic calculus of symmetric Markov processes (in particular a general enough version of Itô's formula), which have been addressed very recently in our separate paper [3]. See Section 2 below for a quick review. 
A special case ( $\widehat{M}=0$ and $M$ purely discontinuous) of the above result was obtained by Chen and Song [5] (see also [1] and [6]) in the broader context of "nearly symmetric" right Markov processes, under somewhat more stringent conditions on $\mu_{\langle M\rangle}$ and $\mu$. In [5] $\mu_{\langle M\rangle}$ is assumed to be in the Kato class while $\mu$ is only assumed to satisfy the condition $\left\|G \mu^{+}\right\|_{\infty}<1$, $G$ being the potential kernel for $X$. Recall that the Revuz measure $\nu$ of a PCAF $A^{\nu}$ is said to be of the Kato class provided

$$
\lim _{t \rightarrow 0}\left\|\mathbf{E} \cdot\left[A_{t}^{\nu}\right]\right\|_{\infty}=0,
$$

and that the Kato class is a subclass of the Hardy class. We write $\mathbf{K}(X)$ for the Kato class and define $\mathbf{K}_{0}(X):=\{\nu \in \mathbf{K}(X) \mid \nu(E)<\infty\}$.

In Section 5, we show that the main result of this paper (Theorem 3.1) yields an extension of the Feynman-Kac formula for zero energy CAF $N^{u}$ studied by Chen and Zhang in [8], where $u$ is a function in $\mathcal{F}$ having Kato class energy measure $\mu_{\left\langle M^{u}\right\rangle}$.

The remainder of the paper is organized as follows. A quick review of the needed stochastic integration with respect to a continuous additive functional of zero energy is given in Section 2 . Section 3 contains the statement of our main result (Theorem 3.1) as well as some auxiliary lemmas needed for its proof. The proof of Theorem 3.1 is completed in section 4 . In Section 5 we show that the Feynman-Kac formula for zero-energy CAF perturbations (Theorem 1.2 of Chen and Zhang [8]) can be deduced from Theorem 3.1 of the present paper.

\section{Stochastic integral for Dirichlet processes}

In this section, we give a quick review of Nakao's [19] definition of stochastic integral with respect to an additive functional of zero energy and our time-reversal approach to the stochastic integral for Dirichlet processes developed in [3].

Let $\left(N(x, d y), H_{t}\right)$ be a Lévy system for $X$; that is, $N(x, d y)$ is a kernel on $\left(E_{\partial}, \mathcal{B}\left(E_{\partial}\right)\right)$ and $H_{t}$ is a PCAF with bounded 1-potential such that for any nonnegative Borel function $\phi$ on $E_{\partial} \times E_{\partial}$ vanishing on the diagonal and any $x \in E_{\partial}$,

$$
\mathbf{E}_{x}\left[\sum_{s \leq t} \phi\left(X_{s-}, X_{s}\right)\right]=\mathbf{E}_{x}\left[\int_{0}^{t} \int_{E_{\partial}} \phi\left(X_{s}, y\right) N\left(X_{s}, d y\right) d H_{s}\right] .
$$

To simplify notation, we will write

$$
N \phi(x):=\int_{E_{\partial}} \phi(x, y) N(x, d y)
$$

and

$$
(N \phi * H)_{t}:=\int_{0}^{t} N \phi\left(X_{s}\right) d H_{s}
$$

Let $\mu_{H}$ be the Revuz measure of the PCAF $H$. Then the jumping measure $J$ and the killing measure $\kappa$ of $X$ are given by

$$
J(d x, d y)=\frac{1}{2} \mu_{H}(d x) N(x, d y) 1_{E}(y), \quad \text { and } \quad \kappa(d x)=N(x,\{\partial\}) \mu_{H}(d x) .
$$


These measures feature in the Beurling-Deny decomposition of $\mathcal{E}:$ for $f, g \in \mathcal{F}$,

$$
\mathcal{E}(f, g)=\mathcal{E}^{(c)}(f, g)+\int_{E \times E}(f(x)-f(y))(g(x)-g(y)) J(d x, d y)+\int_{E} f(x) g(x) \kappa(d x),
$$

where $\mathcal{E}^{(c)}$ is the strongly local part of $\mathcal{E}$.

For $u \in \mathcal{F}$, the martingale part $M_{t}^{u}$ in (1.5) can be decomposed as

$$
M_{t}^{u}=M_{t}^{u, c}+M_{t}^{u, j}+M_{t}^{u, \kappa} \quad \text { for every } t \in\left[0, \infty\left[, \quad \mathbf{P}_{x^{-}} \text {a.s. for } \mathcal{E} \text {-q.e. } x \in E,\right.\right.
$$

where $M_{t}^{u, c}$ is the continuous part of martingale $M^{u}$, and

$$
\begin{aligned}
M_{t}^{u, j}= & \lim _{\varepsilon \downarrow 0}\left\{\sum_{0<s \leq t}\left(u\left(X_{s}\right)-u\left(X_{s-}\right)\right) 1_{\left\{\left|u\left(X_{s}\right)-u\left(X_{s-}\right)\right|>\varepsilon\right\}} 1_{\{s<\zeta\}}\right. \\
& \left.-\int_{0}^{t}\left(\int_{\left\{y \in E:\left|u(y)-u\left(X_{s}\right)\right|>\varepsilon\right\}}\left(u(y)-u\left(X_{s}\right)\right) N\left(X_{s}, d y\right)\right) d H_{s}\right\}, \\
M_{t}^{u, \kappa}= & \int_{0}^{t} u\left(X_{s}\right) N\left(X_{s},\{\partial\}\right) d H_{s}-u\left(X_{\zeta-}\right) 1_{\left\{t \geq \zeta_{i}\right\}},
\end{aligned}
$$

are the jump and killing parts of $M^{u}$, respectively. See Theorem A.3.9 of [11]. The limit in the expression for $M^{u, j}$ is in the sense of convergence in the norm of $\stackrel{\circ}{\mathcal{M}}$ and of convergence in probability under $\mathbf{P}_{x}$ for $\mathcal{E}$-q.e. $x \in E$ (see [11]). The Revuz measure $\mu_{\left\langle M^{u}\right\rangle}$ of $\left\langle M^{u}\right\rangle$ will usually be denoted by $\mu_{\langle u\rangle}$.

Let $\mathcal{N}^{*} \subset \mathcal{N}$ denote the class of continuous additive functionals of the form $N^{u}+\int_{0}^{\cdot} g\left(X_{s}\right) d s$ for some $u \in \mathcal{F}$ and $g \in L^{2}(E ; m)$. Nakao [19] constructed a certain linear map $\Gamma$ from $\mathcal{M}$ into $\mathcal{N}^{*}$ in the following way. It is shown in [19] that, for every $Z \in \stackrel{\circ}{\mathcal{M}}$, there is a unique $w \in \mathcal{F}$ such that

$$
\mathcal{E}_{1}(w, f)=\frac{1}{2} \mu_{\left\langle M^{f}+M^{f, \kappa}, Z\right\rangle}(E) \quad \text { for every } f \in \mathcal{F} .
$$

This unique $w$ is denoted by $\gamma(Z)$. The operator $\Gamma$ is defined by

$$
\Gamma(Z)_{t}=N_{t}^{\gamma(Z)}-\int_{0}^{t} \gamma(Z)\left(X_{s}\right) d s \quad \text { for } Z \in \stackrel{\circ}{\mathcal{M}}
$$

It is shown in Nakao [19] that $\Gamma(Z)$ can be characterized by the following equation

$$
\lim _{t \downarrow 0} \frac{1}{t} \mathbf{E}_{g \cdot m}\left[\Gamma(Z)_{t}\right]=-\frac{1}{2} \mu_{\left\langle M^{g}+M^{g, \kappa}, Z\right\rangle}(E) \quad \text { for every } g \in \mathcal{F}_{b} .
$$

So in particular we have $\Gamma\left(M^{u}\right)=N^{u}$ for $u \in \mathcal{F}$. Nakao [19] used the operator $\Gamma$ to define a stochastic integral

$$
\int_{0}^{t} f\left(X_{s}\right) d N_{s}^{u}:=\Gamma\left(f * M^{u}\right)_{t}-\frac{1}{2}\left\langle M^{f, c}+M^{f, j}, M^{u, c}+M^{u, j}\right\rangle_{t},
$$


where $u \in \mathcal{F}, f \in \mathcal{F} \cap L^{2}\left(E ; \mu_{\langle u\rangle}\right)$ and $\left(f * M^{u}\right)_{t}:=\int_{0}^{t} f\left(X_{s-}\right) d M_{s}^{u}$. If we define

$$
\widetilde{\mathcal{N}}:=\left\{N \in \mathcal{N} \mid N=N^{u}+A^{\mu} \text { for some } u \in \mathcal{F} \text { and some signed smooth measure } \mu\right\},
$$

then we see by $(2.2)$ that $\int_{0}^{\cdot} f\left(X_{s}\right) d N_{s}^{u} \in \tilde{\mathcal{N}}$ if $u \in \mathcal{F}$ and $f \in \mathcal{F} \cap L^{2}\left(E ; \mu_{\langle u\rangle}\right)$. However Nakao's definition of stochastic integral places restrictions on the integrand $f\left(X_{t}\right)$ and on the integrator $N^{u}$ that are too stringent for our study of the perturbation theory of general symmetric Markov processes. We now recall the definition of the stochastic integral introduced in our recent paper [3], using time-reversal.

For $T$ an $\left(\mathcal{F}_{t}\right)$-stopping time, we will use $T_{p}$ and $T_{i}$ to denote, respectively, the predictable and totally inaccessible parts of the given $\left(\mathcal{F}_{t}\right)$-stopping time $T$ of $X$, that is, $T_{p}:=T_{\Lambda_{p}}$ and $T_{i}:=T_{\Lambda_{i}}$, where $\Lambda_{p}:=\left\{T<\infty, X_{T-}=X_{T}\right\}, \Lambda_{i}:=\left\{T<\infty, X_{T-} \in E, X_{T-} \neq X_{T}\right\}$ (see Theorem 44.5 in M. Sharpe [20]). It is shown in [20] that $T_{p}$ and $T_{i}$ are $\left(\mathcal{F}_{t}\right)$-stopping times if $T$ is an $\left(\mathcal{F}_{t}\right)$-stopping time. We set $I(T):=\llbracket 0, T \llbracket \cup \llbracket T_{i} \rrbracket$.

For a locally square-integrable MAF $M_{t}$ on $I(\zeta)$, it is shown in [3] (cf. [2, Lemma 3.2]) that there is a Borel function $\varphi$ on $\left(E \times E_{\partial}\right) \cup(\{\partial\} \times\{\partial\})$ with $\varphi(x, x)=0$ for all $x \in E_{\partial}$ so that

$$
\left.M_{t}-M_{t-}=\varphi\left(X_{t-}, X_{t}\right) \quad \text { for every } t \in\right] 0, \zeta_{p}\left[, \mathbf{P}_{m^{-}}\right. \text {a.s. }
$$

Such a function $\varphi$ is unique up to a measure $J^{*}$-null set on $E \times E_{\partial}$, where $J^{*}$ denotes the measure $\frac{1}{2} N(x, d y) \mu_{H}(d x)$ on $E \times E_{\partial}$. We will call $\varphi$ the jump function of $M$.

Definition 2.1 Let $M$ be a locally square-integrable MAF on $I(\zeta)$ with jump function $\varphi$. Assume

$$
\int_{0}^{t} \int_{E}\left(\widehat{\varphi}^{2} 1_{\{|\widehat{\varphi}| \leq 1\}}+|\widehat{\varphi}| 1_{\{|\widehat{\varphi}|>1\}}\right)\left(X_{s}, y\right) N\left(X_{s}, d y\right) d H_{s}<\infty \quad \text { for every } t<\zeta, \quad \mathbf{P}_{x^{-} \text {-a.s. }}
$$

for $\mathcal{E}$-q.e. $x \in E$, where $\widehat{\varphi}(x, y):=\varphi(x, y)+\varphi(y, x)$. Define, $\mathbf{P}_{m}$-a.s. on $[0, \zeta[$,

$$
\Lambda(M)_{t}:=-\frac{1}{2}\left(M_{t}+M_{t} \circ r_{t}+\varphi\left(X_{t}, X_{t-}\right)+K_{t}\right) \quad \text { for } t \in[0, \zeta[,
$$

where $K_{t}$ is the purely discontinuous local MAF on $I(\zeta)$ with

$$
K_{t}-K_{t-}=-\widehat{\varphi}\left(X_{t-}, X_{t}\right) \quad \text { for } t<\zeta, \quad \mathbf{P}_{x^{-}} \text {a.s. for } \mathcal{E} \text {-q.e. } x \in E .
$$

It is shown in [3, Theorem 3.5] that $\Lambda(M)=\Gamma(M)$ when $M$ is an MAF of $X$ having finite energy. In other words, the above $\Lambda$ operator extends Nakao's $\Gamma$ operator.

Note that for $f \in \mathcal{F}_{\text {loc }}, M^{f, c}$ is well defined as a continuous MAF on [0, $\zeta$ [ locally of finite energy. Moreover, for $f \in \mathcal{F}_{\text {loc }}$ and a locally square-integrable MAF $M$ on $I(\zeta)$,

$$
t \mapsto(f * M)_{t}:=\int_{0}^{t} f\left(X_{s-}\right) d M_{s}
$$

is a locally square-integrable MAF on $I(\zeta)$. For a locally square-integrable MAF $M$ on $I(\zeta)$, denote by $M^{c}$ its continuous part, which is also a locally square-integrable MAF on $I(\zeta)$ (see Theorem 8.23 in [12]). The following definition of stochastic integral is introduced in [3]. 
Definition 2.2 (Stochastic integral) Suppose that $M$ is a locally square-integrable MAF on $I(\zeta)$ and $f \in \mathcal{F}_{\text {loc }}$. Let $\varphi: E_{\partial} \times E_{\partial} \rightarrow \mathbb{R}$ be a jump function for $M$, and assume that $\varphi$ satisfies condition (2.5). Define, $\mathbf{P}_{m}$-a.s. on $[0, \zeta[$,

$$
\begin{array}{rl}
\int_{0}^{t} & f\left(X_{s-}\right) d \Lambda(M)_{s} \\
& :=\Lambda(f * M)_{t}-\frac{1}{2}\left\langle M^{f, c}, M^{c}\right\rangle_{t}+\frac{1}{2} \int_{0}^{t} \int_{E}\left(f(y)-f\left(X_{s}\right)\right) \varphi\left(y, X_{s}\right) N\left(X_{s}, d y\right) d H_{s}
\end{array}
$$

whenever $\Lambda(f * M)$ is well defined and the third term in the right hand side of (2.8) is absolutely convergent.

It is shown in [3, Remark 3.8(ii) and Theorem 4.6] that the above defined stochastic integral extends Nakao's definition of the stochastic integral (2.4) and enjoys a generalized Itô formula.

\section{Perturbation}

Recall that a smooth measure $\mu$ is in the Hardy class (write $\mu \in \mathbf{H}(X)$ ) if there are constants $\delta \in] 0, \infty[$ and $\gamma \in[0, \infty[$ such that

$$
\int_{E} u^{2} d \mu \leq \delta \mathcal{E}(u, u)+\gamma \int_{E} u^{2} d m \quad \text { for } u \in \mathcal{F} .
$$

A well-known sufficient condition for $\mu \in \mathbf{H}(X)$ is that for some $\delta>0$ and $\beta \geq 0$ the $\beta$-potential $U^{\beta} \mu$ is bounded above $\mathcal{E}$-q.e. by $\delta$, in which case $\gamma=\delta \beta$ does the job in (3.1).

Let $M, \widehat{M}$ be two locally square-integrable MAFs on $I(\zeta)$. Let $M^{c}$ and $\widehat{M}^{c}$ denote the continuous parts of $M$ and $\widehat{M}$ respectively, and let $\varphi$ and $\psi$ be jump functions for $M$ and $\widehat{M}$ respectively; thus $\varphi$ and $\psi$ are Borel functions on $E_{\partial} \times E_{\partial}$, vanishing on the diagonal, such that

$$
\left.M_{t}-M_{t-}=\varphi\left(X_{t-}, X_{t}\right) \quad \text { and } \quad \widehat{M}_{t}-\widehat{M}_{t-}=\psi\left(X_{t-}, X_{t}\right) \quad \text { for } t \in\right] 0, \zeta_{p}\left[\quad \mathbf{P}_{m}\right. \text {-a.s. }
$$

We assume $\varphi>-1$ and $\psi>-1$ on $E_{\partial} \times E_{\partial}$. Let $\langle M\rangle$ and $\langle\widehat{M}\rangle$ denote the dual predictable projections of $[M]$ and $[\widehat{M}]$ respectively. Note that

$$
\langle M\rangle_{t}=\left\langle M^{c}\right\rangle_{t}+\int_{0}^{t} \int_{E_{\partial}} \varphi\left(X_{s}, y\right)^{2} N\left(X_{s}, d y\right) d H_{s}, \quad t<\zeta,
$$

and

$$
\langle\widehat{M}\rangle_{t}=\left\langle\widehat{M}^{c}\right\rangle_{t}+\int_{0}^{t} \int_{E_{\partial}} \psi\left(X_{s}, y\right)^{2} N\left(X_{s}, d y\right) d H_{s}, \quad t<\zeta .
$$

Let $\mu$ be a signed smooth measure; thus $\mu$ uniquely determines a continuous additive functional $A^{\mu}$ of bounded variation on each compact time interval. Let $\mu_{\langle M\rangle}$ and $\mu_{\langle\widehat{M}\rangle}$ be the smooth measures associated with the PCAFs $\langle M\rangle_{t}$ and $\langle\widehat{M}\rangle_{t}$. Then

$$
\mu_{\langle M\rangle}=\mu_{\left\langle M^{c}\right\rangle}+N\left(\varphi^{2}\right) \mu_{H} \quad \text { and } \quad \mu_{\langle\widehat{M}\rangle}=\mu_{\left\langle\widehat{M}^{c}\right\rangle}+N\left(\psi^{2}\right) \mu_{H}
$$


We assume $\mu_{\langle M\rangle}, \mu_{\langle\widehat{M}\rangle}$ and $|\mu|$ are in $\mathbf{H}(X)$. Let $\delta\left(\mu_{\langle M\rangle}\right), \delta\left(\mu_{\langle\widehat{M}\rangle}\right), \delta\left(\mu^{+}\right), \delta\left(\varphi^{2}\right)$ and $\delta\left(\psi^{2}\right)$ denote the coefficient of $\mathcal{E}(u)$ and $\gamma\left(\mu_{\langle M\rangle}\right), \gamma\left(\mu_{\langle\widehat{M}\rangle}\right), \gamma\left(\mu^{+}\right), \gamma\left(\varphi^{2}\right)$ and $\gamma\left(\psi^{2}\right)$ the coefficient of $\|u\|_{2}^{2}$ in the estimate (3.1) for $\mu_{\langle M\rangle}, \mu_{\langle\widehat{M}\rangle}, \mu^{+}, N\left(1_{E \times E} \cdot \varphi^{2}\right) \mu_{H}$ and $N\left(1_{E \times E} \cdot \psi^{2}\right) \mu_{H}$, respectively. We define

$$
\delta_{0}:=\sqrt{2 \delta\left(\mu_{\langle M\rangle}\right)}+\sqrt{2 \delta\left(\mu_{\langle\widehat{M}\rangle}\right)}+\delta\left(\mu^{+}\right)+\sqrt{\delta\left(\varphi^{2}\right) \delta\left(\psi^{2}\right)} .
$$

Given these elements, we define a quadratic form $\mathcal{Q}$ on $\mathcal{F}$ : For $f, g \in \mathcal{F}$,

$$
\begin{aligned}
\mathcal{Q}(f, g):=\mathcal{E}(f, g) & -\int_{E} g d \mu_{\left\langle M^{f}, M\right\rangle}-\int_{E} f d \mu_{\left\langle M^{g}, \widehat{M}\right\rangle}-\int_{E} f g d \mu \\
& -\int_{E \times E} f(y) g(x) \varphi(x, y) \psi(y, x) N(x, d y) \mu_{H}(d x) .
\end{aligned}
$$

It is easy to check that there is a constant $C>0$ that

$$
|\mathcal{Q}(f, g)| \leq C \mathcal{E}_{1}(f, f)^{1 / 2} \mathcal{E}_{1}(g, g)^{1 / 2}, \quad f, g \in \mathcal{F} .
$$

Moreover,

$$
\mathcal{Q}_{\alpha}(f, f):=\mathcal{Q}(f, f)+\alpha\|f\|_{2}^{2} \geq\left(1-\delta_{0}\right) \mathcal{E}(f, f)+\left(\alpha-\alpha_{0}\right)\|f\|_{2}^{2}, \quad f \in \mathcal{F},
$$

where

$$
\begin{aligned}
& \alpha_{0}:=\gamma\left(\mu_{\langle M\rangle}\right) \sqrt{2 / \delta\left(\mu_{\langle M\rangle}\right)}+\gamma\left(\mu_{\langle\widehat{M}\rangle}\right) \sqrt{2 / \delta\left(\mu_{\langle\widehat{M}\rangle}\right)} \\
&+\gamma\left(\mu^{+}\right)+\sqrt{\delta\left(\varphi^{2}\right) \delta\left(\psi^{2}\right)}\left\{\frac{\gamma\left(\varphi^{2}\right)}{\delta\left(\varphi^{2}\right)} \vee \frac{\gamma\left(\psi^{2}\right)}{\delta\left(\psi^{2}\right)}\right\} .
\end{aligned}
$$

The quadratic form $(\mathcal{Q}, \mathcal{F})$ is closed on $L^{2}(E ; m)$. Standard resolvent theory now yields the existence of the associated strongly continuous semigroup $\left(Q_{t}\right)_{t \geq 0}$ of operators on $L^{2}(E ; m)$ with $\left\|Q_{t}\right\|_{2 \rightarrow 2} \leq$ $e^{\alpha_{0} t}$ for all $t \geq 0$.

Define a multiplicative functional $Z=\left(Z_{t}\right)$ by

$$
Z_{t}:=\operatorname{Exp}\left(\widehat{M}_{t}\right) \circ r_{t} \operatorname{Exp}\left(M_{t}+A_{t}^{\mu}+\left\langle M^{c}, \widehat{M}^{c}\right\rangle_{t}\right)\left(1+\psi\left(X_{t}, X_{t-}\right)\right)
$$

and an operator

$$
T_{t} f(x):=\mathbf{E}_{x}\left[Z_{t} f\left(X_{t}\right)\right] .
$$

The main result of this paper is the following. Because of (3.5), the condition (3.8) below holds if the constant $\delta_{0}$ defined in (3.2) is strictly less than 1 .

Theorem 3.1 Assume that $\mu_{\langle M\rangle}, \mu_{\langle\widehat{M}\rangle}$ and $|\mu|$ are all in the Hardy class $\mathbf{H}(X)$, and that there are constants $\alpha>0$ and $c>1$ such that

$$
c^{-1} \mathcal{E}_{1}(u, u) \leq \mathcal{Q}_{\alpha}(u, u) \leq c \mathcal{E}_{1}(u, u) \quad \text { for } u \in \mathcal{F}_{b} .
$$

Then $\left\{T_{t}, t \geq 0\right\}$ defined by (3.7) coincides with the strongly continuous semigroup $\left\{Q_{t}, t \geq 0\right\}$ on $L^{2}(E ; m)$ associated with $(\mathcal{Q}, \mathcal{F})$. 
The rest of this section is devoted to the statement and proof of two lemmas needed for the proof of Theorem 3.1.

Lemma 3.2 (i) If $\mu_{\left\langle M^{c}\right\rangle}, \mu_{\left\langle\widehat{M}^{c}\right\rangle},|\mu|, N(|\varphi|) \mu_{H}$ and $N(|\psi|) \mu_{H}$ are measures in $\mathbf{K}(X)$, then the semigroup $\left\{T_{t}, t \geq 0\right\}$ defined by (3.7) is a semigroup of bounded linear operators in $L^{2}(E ; m)$.

(ii) Let $F$ be a closed set and $G$ its fine interior under $X$. If

$$
1_{F}\left(\mu_{\left\langle M^{c}\right\rangle}+\mu_{\left\langle\widehat{M}^{c}\right\rangle}+|\mu|+N(|\varphi|) \mu_{H}+N(|\psi|) \mu_{H}\right) \in \mathbf{K}(X)
$$

and if $\Lambda\left(\widehat{M}^{c}\right)_{t}=N_{t}^{\rho}-\int_{0}^{t} \rho\left(X_{s}\right) d s \mathbf{P}_{m}$-a.s. on $\left\{t<\tau_{G}\right\}$ for some $\rho \in \mathcal{F}$ bounded on $G$ such that $1_{F} \mu_{\langle\rho\rangle} \in \mathbf{K}(X)$, then there exists a constant $k>0$ such that for non-negative $f, g \in L^{2}(G ; m)$

$$
\mathbf{E}_{m}\left[f\left(X_{t}\right) g\left(X_{0}\right) \sup _{s \in\left[0, t \wedge \tau_{G}[\right.} Z_{s}\right] \leq k e^{k t}\|f\|_{2}\|g\|_{2} \quad \text { for } t \geq 0 .
$$

Proof. (i): Since $\log (1+t) \leq t^{+}(:=t \vee 0)$, for $t<\zeta$

$$
\begin{aligned}
\operatorname{Exp}\left(M_{t}\right) & =\exp \left(M_{t}^{c}-\frac{1}{2}\left\langle M^{c}\right\rangle_{t}+M_{t}^{d}+\sum_{0<s \leq t}\left(\log \left(1+\varphi\left(X_{s-}, X_{s}\right)\right)-\varphi\left(X_{s-}, X_{s}\right)\right)\right) \\
& =\exp \left(M_{t}^{c}-\frac{1}{2}\left\langle M^{c}\right\rangle_{t}-\int_{0}^{t} N(\varphi)\left(X_{s}\right) d H_{s}+\sum_{0<s \leq t} \log \left(1+\varphi\left(X_{s-}, X_{s}\right)\right)\right) \\
& \leq \exp \left(M_{t}^{c}-\frac{1}{2}\left\langle M^{c}\right\rangle_{t}+\int_{0}^{t} N\left(\varphi^{-}\right)\left(X_{s}\right) d H_{s}+\sum_{0<s \leq t} \varphi^{+}\left(X_{s-}, X_{s}\right)\right) .
\end{aligned}
$$

where we use the fact that $m_{t}:=\sum_{0<s \leq t} \varphi\left(X_{s-}, X_{s}\right)-\int_{0}^{t} N(\varphi)\left(X_{s}\right) d H_{s}$ is a purely discontinuous martingale and coincides with $M_{t}^{d}$ because $N(|\varphi|) \mu_{H} \in \mathbf{K}(X)$. Similarly

$$
\begin{aligned}
& \operatorname{Exp}\left(\widehat{M}_{t}\right) \circ r_{t} \cdot\left(1+\bar{\psi}\left(X_{t-}, X_{t}\right)\right) \\
& \quad=\exp \left(\widehat{M}_{t}^{c} \circ r_{t}-\frac{1}{2}\left\langle\widehat{M}^{c}\right\rangle_{t}-\int_{0}^{t} N(\psi)\left(X_{s}\right) d H_{s}+\sum_{0<s \leq t} \log \left(1+\bar{\psi}\left(X_{s-}, X_{s}\right)\right)\right) \\
& \quad \leq \exp \left(\widehat{M}_{t}^{c} \circ r_{t}-\frac{1}{2}\left\langle\widehat{M}^{c}\right\rangle_{t}+\int_{0}^{t} N\left(\psi^{-}\right)\left(X_{s}\right) d H_{s}+\sum_{0<s \leq t} \bar{\psi}^{+}\left(X_{s-}, X_{s}\right)\right),
\end{aligned}
$$

where $\bar{\psi}(x, y):=\psi(y, x), \bar{\psi}^{+}(x, y):=\psi^{+}(y, x)$. Decompose the CAF $A^{\mu}$ as the difference $A^{\mu^{+}}-A^{\mu^{-}}$ of PCAFs with mutually singular Revuz measures $\mu^{+}$and $\mu^{-}$, respectively. Then $\mu^{+} \leq|\mu|$, and because $\mu_{\left\langle M^{c}\right\rangle}+\mu_{\left\langle\widehat{M}^{c}\right\rangle}+|\mu|+N(|\varphi|) \mu_{H}+N(|\psi|) \mu_{H} \in \mathbf{K}(X)$, so also

$$
\eta:=\frac{9}{2}\left(\mu_{\left\langle M^{c}\right\rangle}+\mu_{\left\langle\widehat{M}^{c}\right\rangle}\right)+3 \mu^{+}+3 N\left(\varphi^{-}\right) \mu_{H}+3 N\left(\psi^{-}\right) \mu_{H} \in \mathbf{K}(X) .
$$


Let $f$ and $g$ be non-negative elements of $L^{2}(E ; m)$. Then by Hölder's inequality and the expression (3.6) for $Z_{t}$,

$$
\begin{aligned}
\mathbf{E}_{m}\left[g\left(X_{0}\right) Z_{t} f\left(X_{t}\right)\right] \leq \mathbf{E}_{m}\left[g\left(X_{0}\right)^{2} e^{3 M_{t}^{c}-(9 / 2)\left\langle M^{c}\right\rangle_{t}}\right]^{1 / 3} \\
\\
\times \mathbf{E}_{m}\left[f\left(X_{t}\right)^{2} e^{3 \widehat{M}_{t}^{c} \text { or } r_{t}-(9 / 2)\left\langle\widehat{M}^{c}\right\rangle_{t}}\right]^{1 / 3} \mathbf{E}_{m}\left[g\left(X_{0}\right) e^{B_{t}+D_{t}} f\left(X_{t}\right)\right]^{1 / 3}
\end{aligned}
$$

where $D_{t}:=3 \sum_{0<s \leq t} \bar{\psi}^{+}\left(X_{s-}, X_{s}\right)$ and

$$
B_{t}:=\frac{3}{2}\left\langle M^{c}\right\rangle_{t}+\frac{3}{2}\left\langle\widehat{M}^{c}\right\rangle_{t}+3 A_{t}^{\mu^{+}}+3 \int_{0}^{t} N\left(\varphi^{-}+\psi^{-}\right)\left(X_{s}\right) d H_{s}+3 \sum_{0<s \leq t} \varphi^{+}\left(X_{s-}, X_{s}\right)
$$

is the sum of the PCAF associated with the Revuz measure $\eta$ and the discontinuous increasing AF $3 \sum_{0<s<t} \varphi^{+}\left(X_{s-}, X_{s}\right)$. Note that $\widehat{D}_{t}:=3 \sum_{0<s<t} \psi^{+}\left(X_{s-}, X_{s}\right)=D_{t} \circ r_{t} \mathbf{P}_{m^{-}}$a.s. on $\{t<\zeta\}$. Now $e^{3 M_{t}^{c}-(9 / 2)\left\langle M^{c}\right\rangle_{t}}$ is a positive supermartingale, so $\mathbf{E}_{x}\left[e^{3 M_{t}^{c}-(9 / 2)\left\langle M^{c}\right\rangle_{t}}\right] \leq 1$ for $\mathcal{E}$-q.e. $x \in E$. Thus the first factor on the right side of (3.11) is no bigger than $\|g\|_{2}^{2 / 3}$. Because $\left\langle\widehat{M}^{c}\right\rangle$ is even (i.e., $\left\langle\widehat{M}^{c}\right\rangle_{t} \circ r_{t}=\left\langle\widehat{M}^{c}\right\rangle_{t}, \mathbf{P}_{m^{-}}$-a.s. on $\{t<\zeta\}$ for each $t>0$; see (3.13) in [9]), the middle factor on the right side of (3.11) is equal to

$$
\mathbf{E}_{m}\left[f\left(X_{t}\right)^{2} e^{3 \widehat{M}_{t}^{c} \circ r_{t}-(9 / 2)\left\langle\widehat{M}^{c}\right\rangle_{t} \circ r_{t}}\right]^{1 / 3}=\mathbf{E}_{m}\left[f\left(X_{0}\right)^{2} e^{3 \widehat{M}_{t}^{c}-(9 / 2)\left\langle\widehat{M}^{c}\right\rangle_{t}}\right]^{1 / 3} \leq\|f\|_{2}^{2 / 3},
$$

because $e^{3 \widehat{M}_{t}^{c}-(9 / 2)\left\langle\widehat{M}^{c}\right\rangle_{t}}$ is also a positive supermartingale. Finally, by Proposition 2.3 in Chen and Song [5], the cube of the last factor in (3.11) is estimated by

$$
\begin{aligned}
\mathbf{E}_{g m}\left[e^{2 B_{t}} f\left(X_{t}\right)\right]^{1 / 2} \mathbf{E}_{g m}\left[e^{2 D_{t}} f\left(X_{t}\right)\right]^{1 / 2} & =\mathbf{E}_{m}\left[e^{2 B_{t}} f\left(X_{t}\right) g\left(X_{0}\right)\right]^{1 / 2} \mathbf{E}_{m}\left[e^{2 D_{t} \circ r_{t}} f\left(X_{0}\right) g\left(X_{t}\right)\right]^{1 / 2} \\
& \leq\left\|\mathbf{E} \cdot\left[e^{4 B_{t}}\right]\right\|_{\infty}^{1 / 4}\left\|\mathbf{E} \cdot\left[e^{4 \widehat{D}_{t}}\right]\right\|_{\infty}^{1 / 4}\|f\|_{2}\|g\|_{2} \\
& \leq k_{0} e^{k_{0} t}\|f\|_{2}\|g\|_{2}
\end{aligned}
$$

for some $k_{0}>0$. Feeding these estimates into (3.11) we find that

$$
\mathbf{E}_{m}\left[f\left(X_{t}\right) Z_{t} g\left(X_{0}\right)\right] \leq k_{0}^{1 / 3} \cdot e^{k_{0} t / 3}\|f\|_{2}\|g\|_{2},
$$

which proves the assertion.

(ii) By (3.9) and (3.10), we have that $\mathbf{P}_{m}$-a.s. on $\left\{t<\tau_{G}\right\}$

$$
\begin{aligned}
Z_{t} \leq & \exp \left(-2 \Lambda\left(\widehat{M}^{c}\right)_{t}\right) \operatorname{Exp}\left(M_{t}^{c}-\widehat{M}_{t}^{c}\right) \\
& \times \exp \left(\sum_{0<s \leq t}\left(\varphi^{+}+\bar{\psi}^{+}\right)\left(X_{s-}, X_{s}\right)\right) \exp \left(A_{t}^{\mu}+\int_{0}^{t} N\left(\varphi^{-}+\psi^{-}\right)\left(X_{s}\right) d H_{s}\right) \\
= & \exp \left(2 \rho\left(X_{0}\right)-2 \rho\left(X_{t}\right)+2 \int_{0}^{t} \rho\left(X_{s}\right) d s+2 M_{t}^{\rho}\right) \operatorname{Exp}\left(M_{t}^{c}-\widehat{M}_{t}^{c}\right) \\
& \times \exp \left(\sum_{0<s \leq t}\left(\varphi^{+}+\bar{\psi}^{+}\right)\left(X_{s-}, X_{s}\right)\right) \exp \left(A_{t}^{\mu}+\int_{0}^{t} N\left(\varphi^{-}+\psi^{-}\right)\left(X_{s}\right) d H_{s}\right) .
\end{aligned}
$$


Thus, $\mathbf{P}_{m}$-a.s. on $\left\{t<\tau_{G}\right\}$

$$
\begin{gathered}
\sup _{0 \leq s<t} Z_{s} \leq \exp \left[(4+2 t)\|\rho\|_{G, \infty}\right] \sup _{0 \leq s<t} \exp \left[2\left(1_{F} * M^{\rho}\right)_{s}\right] \\
\times \sup _{0 \leq s<t} \exp \left(\left(1_{F} * K^{c}\right)_{s}-\frac{1}{2}\left\langle 1_{F} * K^{c}\right\rangle_{s}\right) \\
\times \exp \left(\sum_{0<s \leq t} 1_{F}\left(X_{s-}\right) \bar{\psi}^{+}\left(X_{s-}, X_{s}\right)\right) \\
\times \exp \left(\left(1_{F} A^{\mu^{+}}\right)_{t}+\int_{0}^{t} 1_{F}\left(X_{s}\right) N\left(\varphi^{-}+\psi^{-}\right)\left(X_{s}\right) d H_{s}+\sum_{0<s \leq t} 1_{F}\left(X_{s-}\right) \varphi^{+}\left(X_{s-}, X_{s}\right)\right),
\end{gathered}
$$

where $K^{c}:=M^{c}-\widehat{M}^{c}$ and $1_{F} * K_{t}^{c}:=\int_{0}^{t} 1_{F}\left(X_{s-}\right) d K_{s}^{c}$ and $\left(1_{F} A^{\mu^{+}}\right)_{t}:=\int_{0}^{t} 1_{F}\left(X_{s}\right) d A_{s}^{\mu^{+}}$. Applying Doob's inequality to the submartingale $\exp \left(1_{F} * M^{\rho}\right)_{t}$ together with Lemma 4.1(i) in Chen and Zhang [8] we see that, because $1_{F} \mu_{\langle\rho\rangle} \in \mathbf{K}(X)$, the expectation of the eighth power of the second factor of (3.14) is estimated by

$$
\begin{aligned}
\left\|\mathbf{E} .\left[\sup _{0 \leq s<t} \exp \left(16\left(1_{F} * M^{\rho}\right)_{s}\right)\right]\right\|_{\infty} & \leq\left(\frac{16}{15}\right)^{16}\left\|\mathbf{E} \cdot\left[\exp \left(16\left(1_{F} * M^{\rho}\right)_{t}\right)\right]\right\|_{\infty} \\
& \leq k_{1} e^{k_{1} t}
\end{aligned}
$$

for some $k_{1}>0$. Since $1_{F} \mu_{\left\langle K^{c}\right\rangle} \in \mathbf{K}(X)$, $\exp \left(\left(1_{F} * K^{c}\right)-\frac{1}{2}\left\langle 1_{F} * K^{c}\right\rangle\right)$ is a martingale. Applying Doob's inequality to $\exp \left(\left(1_{F} * K^{c}\right)-\frac{1}{2}\left\langle 1_{F} * K^{c}\right\rangle\right)$, the expectation of the eighth power of (3.15) is estimated by

$$
\begin{aligned}
& \mathbf{E}_{x}\left[\left|\sup _{0 \leq s \leq t} \exp \left(\left(1_{F} * K^{c}\right)_{s}-\frac{1}{2}\left\langle 1_{F} * K^{c}\right\rangle_{s}\right)\right|^{8}\right] \\
\leq & \left(\frac{8}{7}\right)^{8} \mathbf{E}_{x}\left[\exp \left(8\left(1_{F} * K^{c}\right)_{t}-4\left\langle 1_{F} * K^{c}\right\rangle_{t}\right)\right] \\
\leq & \left(\frac{8}{7}\right)^{8} \mathbf{E}_{x}\left[\exp \left(16\left(1_{F} * K^{c}\right)_{t}-128\left\langle 1_{F} * K^{c}\right\rangle_{t}\right)\right]^{1 / 2} \mathbf{E}_{x}\left[\exp \left(120\left\langle 1_{F} * K^{c}\right\rangle_{t}\right)\right]^{1 / 2} \\
\leq & \left(\frac{8}{7}\right)^{8} \sqrt{k_{2}} e^{\left(k_{2} / 2\right) t}
\end{aligned}
$$

for some $k_{2}>0$, because $\exp \left(16\left(1_{F} * K^{c}\right)_{t}-128\left\langle 1_{F} * K^{c}\right\rangle_{t}\right)$ is a martingale. Noting that $1_{F} N(|\varphi|+$ $|\psi|) \mu_{H} \in \mathbf{K}(X)$, by using Proposition 2.3 in [8] again, the expectations of the eighth power of (3.16) (after time reversion with respect to the part process on $G$ ) and (3.17) are estimated by $k_{3} e^{k_{3} t}$ for some $k_{3}>0$. Denote by $C_{t}^{(1)}, C_{t}^{(2)}, C_{t}^{(3)}$ and $C_{t}^{(4)}$, the second factor of (3.14), (3.15), (3.16) and (3.17) respectively. Then

$\mathbf{E}_{1_{G} m}\left[f\left(X_{t}\right) g\left(X_{0}\right) \sup _{0 \leq s<t} Z_{s}: t<\tau_{G}\right] \leq e^{(4+2 t)\|\rho\|_{G, \infty}}\left(\prod_{i=1}^{4} \mathbf{E}_{1_{G} m}\left[\left|C_{t}^{(i)}\right|^{4} f\left(X_{t}\right) g\left(X_{0}\right): t<\tau_{G}\right]\right)^{1 / 4}$. 
For $i=1,2,4$,

$$
\mathbf{E}_{1_{G} m}\left[\left|C_{t}^{(i)}\right|^{4} f\left(X_{t}\right) g\left(X_{0}\right): t<\tau_{G}\right] \leq\|f\|_{2}\|g\|_{2}\left\|\mathbf{E} .\left[\left|C_{t}^{(i)}\right|^{8}: t<\tau_{G}\right]\right\|_{G, \infty},
$$

while for $i=3$,

$$
\mathbf{E}_{1_{G} m}\left[\left|C_{t}^{(3)}\right|^{4} f\left(X_{t}\right) g\left(X_{0}\right): t<\tau_{G}\right] \leq\|f\|_{2}\|g\|_{2}\left\|\mathbf{E} .\left[\left|C_{t}^{(3)} \circ r_{t}\right|^{8}: t<\tau_{G}\right]\right\|_{G, \infty} .
$$

Here $r_{t}$ is the time reverse operator under the part process on $G$. Therefore we have the desired estimate.

Under the assumptions of Theorem 3.1, it is clear that the bilinear form $(\mathcal{Q}, \mathcal{F})$ is a closed, lower-bounded quadratic form. Therefore there exists a strongly continuous semigroup, $\left\{Q_{t}, t \geq 0\right\}$, associated with $(\mathcal{Q}, \mathcal{F})$. Let $\left(L^{\mathcal{Q}}, \mathcal{D}\left(L^{\mathcal{Q}}\right)\right)$ be its corresponding $L^{2}$-generator. On the other hand, it can be shown that the operators $\left\{T_{t}, t \geq 0\right\}$ defined in (3.7) form a strongly continuous semigroup on $L^{2}(E ; m)$. Denote the $L^{2}$-generator of $\left\{T_{t}, t \geq 0\right\}$ by $(L, \mathcal{D}(L))$.

Lemma 3.3 Suppose $\mu_{\langle M\rangle}+\mu_{\langle\widehat{M}\rangle}+|\mu|+N(|\varphi|) \mu_{H} \in \mathbf{K}(X), \mu_{\langle\widehat{M}\rangle}(E)+\int_{E} N(|\psi|) d \mu_{H}<\infty$ and $-1<c_{1} \leq \varphi, \psi \leq c_{2}<\infty$ for some constants $c_{1}, c_{2}$. Then, for $f \in \mathcal{D}\left(L^{\mathcal{Q}}\right)$

$$
\begin{aligned}
Z_{t} f\left(X_{t}\right)= & f\left(X_{0}\right)+\int_{0}^{t} Z_{s-} d\left(M_{s}^{f}+U_{s}^{f}\right)+\int_{0}^{t} Z_{s-} f\left(X_{s-}\right) d\left(M_{s}^{c}-\widehat{M}_{s}^{c}+W_{s}\right) \\
& +\int_{0}^{t} Z_{s} L^{\mathcal{Q}} f\left(X_{s}\right) d s,
\end{aligned}
$$

where $W$ and $U^{f}$ are purely discontinuous local MAFs on $I(\zeta)$ with

$$
W_{t}-W_{t-}=\varphi\left(X_{t-}, X_{t}\right)+\psi\left(X_{t}, X_{t-}\right)+\varphi\left(X_{t-}, X_{t}\right) \psi\left(X_{t}, X_{t-}\right), \quad t<\zeta
$$

and

$$
U_{t}^{f}-U_{t-}^{f}=\left(f\left(X_{t}\right)-f\left(X_{t-}\right)\right)\left(W_{t}-W_{t-}\right), \quad t<\zeta .
$$

Proof. Putting $\bar{\psi}(x, y):=\psi(y, x)$, we see $\int_{E} N\left(1_{E \times E}|\bar{\psi}|^{2}\right) d \mu_{H}=\int_{E} N\left(1_{E \times E}|\psi|^{2}\right) d \mu_{H}<\infty$ and $\int_{E} N\left(1_{E \times E}|\bar{\psi}|\right) d \mu_{H}=\int_{E} N\left(1_{E \times E}|\psi|\right) d \mu_{H}<\infty$. In view of Theorem 5.1.3 in [11], we have $\mathbf{E}_{x}\left[\int_{0}^{t} N\left(1_{E \times E}\left(|\bar{\psi}|+|\bar{\psi}|^{2}\right)\right)\left(X_{s}\right) d H_{s}\right]<\infty$ for $m$-a.e. $x \in E$ for each $t>0$. On the other hand, $N(|\varphi|) \mu_{H} \in \mathbf{K}(X)$ implies $\mathbf{E}_{x}\left[\int_{0}^{t} N(|\varphi|)\left(X_{s}\right) d H_{s}\right]<\infty$ for $m$-a.e. $x \in E$ for each $t>0$. Thus, we have the purely discontinuous local MAF $W$ (resp. $U^{f}$ ) on $I(\zeta)$ with the property (3.20) (resp. (3.21)). Since $\mu_{\langle\widehat{M}\rangle}(E)<\infty, \widehat{M}$ is an MAF having finite energy and so by (2.2) and [3, Theorem 3.5] there is some $\rho \in \mathcal{F}$ such that

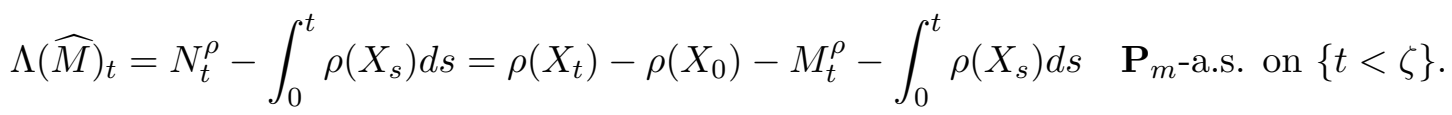

By Definition 2.1,

$$
\widehat{M}_{t} \circ r_{t}=-2 \Lambda(\widehat{M})_{t}-\widehat{M}_{t}-\psi\left(X_{t}, X_{t-}\right)-\widehat{K}_{t} \quad \mathbf{P}_{m} \text {-a.s. on }\{t<\zeta\},
$$


where $\widehat{K}$ is a purely discontinuous local MAF on $I(\zeta)$ with

$$
\widehat{K}_{t}-\widehat{K}_{t-}=-\psi\left(X_{t-}, X_{t}\right)-\psi\left(X_{t}, X_{t-}\right), t<\zeta .
$$

The condition $\int_{E} N(|\psi|) d \mu_{H}=\int_{E} N(|\bar{\psi}|) d \mu_{H} \in \mathbf{K}(X)$ implies the integrability of $\widehat{K}$, hence $\widehat{K}$ is a martingale. Therefore on $\{t<\zeta\}$,

$$
\begin{aligned}
& \operatorname{Exp}\left(\widehat{M}_{t}\right) \circ r_{t}\left(1+\psi\left(X_{t}, X_{t-}\right)\right) \\
= & \left(\exp \left(\widehat{M}_{t}-\frac{1}{2}\left\langle\widehat{M}^{c}\right\rangle_{t}\right) \prod_{0<s \leq t}\left(1+\psi\left(X_{s-}, X_{s}\right)\right) e^{-\psi\left(X_{s-}, X_{s}\right)}\right) \circ r_{t}\left(1+\psi\left(X_{t}, X_{t-}\right)\right) \\
= & \exp \left(\widehat{M}_{t} \circ r_{t}-\frac{1}{2}\left\langle\widehat{M}^{c}\right\rangle_{t}\right)\left(\prod_{0<s<t}\left(1+\psi\left(X_{s}, X_{s-}\right)\right) e^{-\psi\left(X_{s}, X_{s-}\right)}\right)\left(1+\psi\left(X_{t}, X_{t-}\right)\right) \\
= & \exp \left(-2 \Lambda(\widehat{M})_{t}-\widehat{M}_{t}-\widehat{K}_{t}-\frac{1}{2}\left\langle\widehat{M}^{c}\right\rangle_{t}\right) \prod_{0<s \leq t}\left(1+\psi\left(X_{s}, X_{s-}\right)\right) e^{-\psi\left(X_{s}, X_{s-}\right)} \\
= & \exp \left(-2 \Lambda(\widehat{M})_{t}\right) \operatorname{Exp}\left(-\widehat{M}_{t}^{c}\right) \operatorname{Exp}\left(-\widehat{M}_{t}^{d}-\widehat{K}_{t}\right) \\
= & \exp \left(-2 \Lambda(\widehat{M})_{t}\right) \operatorname{Exp}\left(-\widehat{M}_{t}-\widehat{K}_{t}\right) .
\end{aligned}
$$

Note that

$$
W_{t}=M_{t}^{d}-\widehat{M}_{t}^{d}-\widehat{K}_{t}+\sum_{0<s \leq t}(\varphi \cdot \bar{\psi})\left(X_{s-}, X_{s}\right)-\int_{0}^{t} N(\varphi \cdot \bar{\psi})\left(X_{s}\right) d H_{s}, \quad t<\zeta .
$$

Thus

$$
\begin{aligned}
Z_{t} & =\exp \left(-2 \Lambda(\widehat{M})_{t}\right) \operatorname{Exp}\left(-\widehat{M}_{t}-\widehat{K}_{t}\right) \operatorname{Exp}\left(M_{t}+A_{t}^{\mu}+\left\langle M^{c}, \widehat{M}^{c}\right\rangle_{t}\right) \\
& =\exp \left(-2 \Lambda(\widehat{M})_{t}\right) \operatorname{Exp}\left(M_{t}-\widehat{M}_{t}-\widehat{K}_{t}+A_{t}^{\mu}-\left[M^{d}, \widehat{M}^{d}+\widehat{K}\right]_{t}\right) \\
& =\exp \left(-2 \Lambda(\widehat{M})_{t}\right) \operatorname{Exp}\left(M_{t}-\widehat{M}_{t}-\widehat{K}_{t}+A_{t}^{\mu}+\sum_{0<s \leq t}(\varphi \cdot \bar{\psi})\left(X_{s-}, X_{s}\right)\right) \\
& =\exp \left(-2 \Lambda(\widehat{M})_{t}\right) \operatorname{Exp}\left(M_{t}^{c}-\widehat{M}_{t}^{c}+W_{t}+A_{t}^{\mu}+\int_{0}^{t} N(\varphi \cdot \bar{\psi})\left(X_{s}\right) d H_{s}\right) \\
& :=\exp \left(-2 \rho\left(X_{t}\right)\right) Z_{t}^{1}
\end{aligned}
$$

with

$$
\begin{aligned}
Z_{t}^{1}:=\exp & \left(2 \rho\left(X_{0}\right)+2 M_{t}^{\rho}+2 \int_{0}^{t} \rho\left(X_{s}\right) d s\right) \\
& \cdot \operatorname{Exp}\left(M_{t}^{c}-\widehat{M}_{t}^{c}+W_{t}+A_{t}^{\mu}+\int_{0}^{t} N(\varphi \cdot \bar{\psi})\left(X_{s}\right) d H_{s}\right) .
\end{aligned}
$$

Note that

$$
1+W_{t}-W_{t-}=\left(1+\varphi\left(X_{t-}, X_{t}\right)\right)\left(1+\psi\left(X_{t}, X_{t-}\right)\right)>0
$$


Let $f \in \mathcal{D}\left(L^{\mathcal{Q}}\right) \subset \mathcal{F}$. Then

$$
\begin{aligned}
\mathcal{E}(f, g)= & \int_{E}\left(-L^{\mathcal{Q}} f\right)(x) g(x) m(d x)+\int_{E} f(x) \mu_{\left\langle M^{g}, \widehat{M}\right\rangle}(d x)+\int_{E} g(x) \mu_{\left\langle M^{f}, M\right\rangle}(d x) \\
& +\int_{E} f(x) g(x) \mu(d x)+\int_{E} g(x)\left(\int_{E} f(y) \varphi(x, y) \psi(y, x) N(x, d y)\right) \mu_{H}(d x) .
\end{aligned}
$$

Applying (2.3) to the (finite energy) MAF $f * \widehat{M}$ (defined by $(f * \widehat{M})_{t}=\int_{0}^{t} f\left(X_{s-}\right) d \widehat{M}_{s}$ ), we have

$$
\lim _{t \rightarrow 0} \frac{1}{t} \mathbf{E}_{g m}\left[2 \Gamma(f * \widehat{M})_{t}\right]=-\int_{E} f(x) \mu_{\left\langle M^{g}, \widehat{M}\right\rangle}(d x) \quad \text { for every } g \in \mathcal{F}_{b} .
$$

By (3.24)-(3.25) above and Theorem 5.2.4 in [11],

$$
\begin{aligned}
N_{t}^{f}= & \int_{0}^{t} L^{\mathcal{Q}} f\left(X_{s}\right) d s+2 \Gamma(f * \widehat{M})_{t}-\left\langle M^{f}, M\right\rangle_{t}-\int_{0}^{t} f\left(X_{s}\right) d A_{s}^{\mu} \\
& -\int_{0}^{t}\left(\int_{E} f(y) \varphi\left(X_{s}, y\right) \psi\left(y, X_{s}\right) N\left(X_{s}, d y\right)\right) d H_{s}
\end{aligned}
$$

Note that it was shown in $[3$, Remark 3.8(ii)] that $\Gamma(f * \widehat{M})=\Lambda(f * \widehat{M})$ and that by Definition 1.2 ,

$$
\begin{array}{rl}
\Lambda(f * \widehat{M})_{t}=\int_{0}^{t} & f\left(X_{s-}\right) d \Lambda(\widehat{M})_{s}+\frac{1}{2}\left\langle M^{f}, \widehat{M}^{c}\right\rangle_{t} \\
& \quad-\frac{1}{2} \int_{0}^{t} \int_{E}\left(f(y)-f\left(X_{s}\right)\right) \psi\left(y, X_{s}\right) N\left(X_{s}, d y\right) d H_{s} .
\end{array}
$$

It follows from (3.26)-(3.27) that

$$
\begin{aligned}
N_{t}^{f}= & \int_{0}^{t} L^{\mathcal{Q}} f\left(X_{s}\right) d s+2 \int_{0}^{t} f\left(X_{s-}\right) d N_{s}^{\rho}-2 \int_{0}^{t} f\left(X_{s}\right) \rho\left(X_{s}\right) d s+\left\langle M^{f}, \widehat{M}^{c}-M\right\rangle_{t}-\int_{0}^{t} f\left(X_{s}\right) d A_{s}^{\mu} \\
& \left.-\int_{0}^{t}\left(\int_{E}\left(f(y)-f\left(X_{s}\right)\right) \psi\left(y, X_{s}\right)+f(y) \varphi\left(X_{s}, y\right) \psi\left(y, X_{s}\right)\right) N\left(X_{s}, d y\right)\right) d H_{s} .
\end{aligned}
$$

By [3, Theorem 4.2],

$$
\begin{aligned}
& \int_{0}^{t} e^{-2 \rho\left(X_{s-}\right)} d N_{s}^{f}-2 \int_{0}^{t} e^{-2 \rho\left(X_{s-}\right)} f\left(X_{s-}\right) d N_{s}^{\rho} \\
= & \int_{0}^{t} e^{-2 \rho\left(X_{s}\right)} L^{\mathcal{Q}} f\left(X_{s}\right) d s-2 \int_{0}^{t} e^{-2 \rho\left(X_{s}\right)} f\left(X_{s}\right) \rho\left(X_{s}\right) d s \\
& +\int_{0}^{t} e^{-2 \rho\left(X_{s}\right)} d\left\langle M^{f}, \widehat{M}^{c}-M\right\rangle_{s}-\int_{0}^{t} e^{-2 \rho\left(X_{s}\right)} f\left(X_{s}\right) d A_{s}^{\mu} \\
& \left.-\int_{0}^{t} e^{-2 \rho\left(X_{s}\right)}\left(\int_{E}\left(f(y)-f\left(X_{s}\right)\right) \psi\left(y, X_{s}\right)+f(y) \varphi\left(X_{s}, y\right) \psi\left(y, X_{s}\right)\right) N\left(X_{s}, d y\right)\right) d H_{s} .
\end{aligned}
$$


Now by the Itô formula in [3, Theorem 4.7] and the above identity, we have

$$
\begin{aligned}
& e^{-2 \rho\left(X_{t}\right)} f\left(X_{t}\right)-e^{-2 \rho\left(X_{0}\right)} f\left(X_{0}\right) \\
= & \int_{0}^{t} e^{-2 \rho\left(X_{s-}\right)} d\left(M_{s}^{f}+N_{s}^{f}\right)-2 \int_{0}^{t} e^{-2 \rho\left(X_{s-}\right)} f\left(X_{s-}\right) d\left(M_{s}^{\rho}+N_{s}^{\rho}\right) \\
& -2 \int_{0}^{t} e^{-2 \rho\left(X_{s-}\right)} d\left\langle M^{\rho, c}, M^{f, c}\right\rangle_{s}+2 \int_{0}^{t} e^{-2 \rho\left(X_{s-}\right)} f\left(X_{s-}\right) d\left\langle M^{\rho, c}, M^{\rho, c}\right\rangle_{s} \\
& +\sum_{0<s \leq t}^{t}\left[\Delta\left(e^{-2 \rho\left(X_{s}\right)} f\left(X_{s}\right)\right)+2 e^{-2 \rho\left(X_{s-}\right)} f\left(X_{s-}\right) \Delta\left(\rho\left(X_{s}\right)\right)-e^{-2 \rho\left(X_{s-}\right)} \Delta\left(f\left(X_{s}\right)\right)\right] \\
= & \int_{0}^{t} e^{-2 \rho\left(X_{s-}\right)} d M_{s}^{f}-2 \int_{0}^{t} e^{-2 \rho\left(X_{s-}\right)} f\left(X_{s-}\right) d M_{s}^{\rho}+\int_{0}^{t} e^{-2 \rho\left(X_{s}\right)} L^{\mathcal{Q}} f\left(X_{s}\right) d s \\
& -2 \int_{0}^{t} e^{-2 \rho\left(X_{s}\right)} f\left(X_{s}\right) \rho\left(X_{s}\right) d s+\int_{0}^{t} e^{-2 \rho\left(X_{s}\right)} d\left\langle M^{f}, \widehat{M}^{c}-M\right\rangle_{s}-\int_{0}^{t} e^{-2 \rho\left(X_{s}\right)} f\left(X_{s}\right) d A_{s}^{\mu} \\
& \left.-\int_{0}^{t} e^{-2 \rho\left(X_{s}\right)}\left(\int_{E}\left(f(y)-f\left(X_{s}\right)\right) \psi\left(y, X_{s}\right)+f(y) \varphi\left(X_{s}, y\right) \psi\left(y, X_{s}\right)\right) N\left(X_{s}, d y\right)\right) d H_{s} \\
& -2 \int_{0}^{t} e^{-2 \rho\left(X_{s-}\right)} d\left\langle M^{\rho, c}, M^{f, c}\right\rangle_{s}+2 \int_{0}^{t} e^{-2 \rho\left(X_{s-}\right)} f\left(X_{s-}\right) d\left\langle M^{\rho, c}, M^{\rho, c}\right\rangle_{s} \\
& +\sum_{0<s \leq t}\left[\Delta\left(e^{-2 \rho\left(X_{s}\right)} f\left(X_{s}\right)\right)+2 e^{-2 \rho\left(X_{s-}\right)} f\left(X_{s-}\right) \Delta\left(\rho\left(X_{s}\right)\right)-e^{-2 \rho\left(X_{s-}\right)} \Delta\left(f\left(X_{s}\right)\right)\right]
\end{aligned}
$$

which is a semimartingale. Note that $Z_{t}^{1}$ can be rewritten as

$$
Z_{t}^{1}=\operatorname{Exp}\left(M_{t}^{c}-\widehat{M}_{t}^{c}+W_{t}+A_{t}^{\mu}+\int_{0}^{t} N(\varphi \cdot \bar{\psi})\left(X_{s}\right) d H_{s}+2 \int_{0}^{t} \rho\left(X_{s}\right) d s\right) \exp \left(2 \rho\left(X_{0}\right)+M_{t}^{2 \rho}\right) .
$$

By Itô's formula for semimartingales,

$$
\begin{aligned}
& Z_{t}^{1}-Z_{0}^{1} \\
= & \int_{0}^{t} Z_{s-}^{1} d\left(M_{s}^{c}-\widehat{M}_{s}^{c}+W_{s}+2 M_{s}^{\rho}+A_{s}^{\mu}+\int_{0}^{s} N(\varphi \cdot \bar{\psi})\left(X_{u}\right) d H_{u}+2 \int_{0}^{s} \rho\left(X_{u}\right) d u\right) \\
& +2 \int_{0}^{t} Z_{s-}^{1} d\left\langle M^{\rho, c}+M^{c}-\widehat{M}^{c}, M^{\rho, c}\right\rangle_{s}+\sum_{0<s \leq t} Z_{s-}^{1}\left(e^{2 \Delta\left(\rho\left(X_{s}\right)\right)}-1-2 \Delta\left(\rho\left(X_{s}\right)\right)\right) \\
& +\sum_{0<s \leq t} Z_{s-}^{1}\left(\varphi\left(X_{s-}, X_{s}\right)+\psi\left(X_{s}, X_{s-}\right)+\varphi\left(X_{s-}, X_{s}\right) \psi\left(X_{s}, X_{s-}\right)\right)\left(e^{2 \Delta\left(\rho\left(X_{s}\right)\right)}-1\right) .
\end{aligned}
$$

Applying Itô's formula to

$$
Z_{t} f\left(X_{t}\right)=Z_{t}^{1} \cdot\left(e^{-2 \rho\left(X_{t}\right)} f\left(X_{t}\right)\right)
$$

and using (3.28)-(3.29), we get (3.19) after many terms cancel out. This calculation is tedious and must be done with care. It is fairly easy to calculate out the martingale part, the quadratic variation part and continuous additive part while applying Itô's formula. However the calculation of the jump part of $Z_{t}^{1} \cdot\left(e^{-2 \rho\left(X_{t}\right)} f\left(X_{t}\right)\right)$ using Itô's formula can be frustrating. The best way to 
calculate $Z_{t} f\left(X_{t}\right)-Z_{t-} f\left(X_{t-}\right)$ directly is perhaps the following. It follows from (3.22) and the fact that the Doléans-Dade's exponential solves an SDE, that

$$
Z_{t}-Z_{t-}=Z_{t-}\left(W_{t}-W_{t-}\right)
$$

and so

$$
\begin{aligned}
Z_{t} f\left(X_{t}\right)-Z_{t-} f\left(X_{t-}\right) & =Z_{t-}\left(\left(1+W_{t}-W_{t-}\right) f\left(X_{t}\right)-f\left(X_{t-}\right)\right) \\
& =Z_{t-}\left(\left(1+W_{t}-W_{t-}\right) \Delta\left(f\left(X_{t}\right)\right)+f\left(X_{t-}\right)\left(W_{t}-W_{t-}\right)\right) .
\end{aligned}
$$

\section{Proof of Theorem 3.1.}

Lemma 4.1 Under the conditions of Theorem 3.1, the quadratic form $(\mathcal{Q}, \mathcal{F})$ defined in (3.3) possesses the positivity preserving property in the sense of [18].

Proof. By Proposition 1.3(i) in [18], it suffices to show $\mathcal{Q}\left(f^{+}, f^{-}\right) \leq 0$ for $f \in \mathcal{F}$. Let $\mathcal{Q}^{(c)+(k)}$ be the sum of continuous part and killing part of $\mathcal{Q}$ :

$$
\begin{aligned}
\mathcal{Q}^{(c)+(k)}(f, g) & :=\mathcal{E}^{(c)}(f, g)+\mathcal{E}^{(k)}(f, g) \\
& -\int_{E} f(x) \mu_{\left\langle M^{g, c}, \widehat{M}^{c}\right\rangle}(d x)-\int_{E} g(x) \mu_{\left\langle M^{f, c}, M^{c}\right\rangle}(d x)-\int_{E} f(x) g(x) \mu(d x) \\
& +\int_{E} f(x) g(x) \psi(x, \partial) N(x,\{\partial\}) \mu_{H}(d x)+\int_{E} f(x) g(x) \varphi(x, \partial) N(x,\{\partial\}) \mu_{H}(d x) .
\end{aligned}
$$

Then we see $\mathcal{Q}^{(c)+(k)}\left(f^{+}, f^{-}\right)=0$ because $\mu_{\langle f\rangle}^{c}(f=0)=0$ and $\mu_{\left\langle f^{+}\right\rangle}^{c}(f<0)=\mu_{\left\langle f^{-}\right\rangle}^{c}(f>$ $0)=0$, where $\mu_{\langle u\rangle}^{c}:=\mu_{\left\langle M^{u, c\rangle}\right.}$ for $u \in \mathcal{F}$. If we let $\mathcal{Q}^{(j)}(f, g):=\mathcal{Q}(f, g)-\mathcal{Q}^{(c)+(k)}(f, g)=$ $\mathcal{E}^{(j)}(f, g)+2 \int_{E \times E \backslash d}(f(x)-f(y)) g(x) \varphi(x, y) J(d x, d y)+2 \int_{E \times E \backslash d}(g(x)-g(y)) f(x) \psi(x, y) J(d x, d y)$, then $Q^{(j)}\left(f^{+}, f^{-}\right)=-2 \int_{E \times E} f^{+}(y) f^{-}(x)(1+\varphi(x, y))(1+\psi(y, x)) J(d x, d y) \leq 0$.

Lemma 4.2 Let $G$ be a finely open (nearly) Borel subset of $E$ and consider the part space $\left(\mathcal{E}_{G}, \mathcal{F}_{G}\right)$ of $(\mathcal{E}, \mathcal{F})$ on $L^{2}(G ; m)$. Then $\mathcal{Q}$ on $\mathcal{F}_{G}$ has the following expression: For $f, g \in \mathcal{F}_{G}$,

$$
\begin{aligned}
\mathcal{Q}(f, g)= & \mathcal{E}(f, g)-\int_{G} f(x) \mu_{\left\langle M^{g}, \widehat{M}\right\rangle}^{G}(d x)-\int_{G} g(x) \mu_{\left\langle M^{f}, M\right\rangle}^{G}(d x)-\int_{G} f(x) g(x) \mu^{G}(d x) \\
& -\int_{G \times G} f(y) g(x) \varphi(x, y) \psi(y, x) N(x, d y) \mu_{H}(d x),
\end{aligned}
$$

where

$$
\begin{aligned}
\mu_{\left\langle M^{g}, \widehat{M}\right\rangle}^{G}(d x) & :=\mu_{\left\langle M^{g, c}, \widehat{M}^{c}\right\rangle}(d x)+\left(\int_{G_{\partial}}(g(y)-g(x)) \psi(x, y) N(x, d y)\right) \mu_{H}(d x), \\
\mu_{\left\langle M^{f}, M\right\rangle}^{G}(d x) & :=\mu_{\left\langle M^{f, c}, M^{c}\right\rangle}(d x)+\left(\int_{G_{\partial}}(f(y)-f(x)) \varphi(x, y) N(x, d y)\right) \mu_{H}(d x), \\
\mu^{G}(d x) & :=\mu(d x)-\left(\int_{E \backslash G}(1+\varphi(x, y)+\psi(x, y)) N(x, d y)\right) \mu_{H}(d x) .
\end{aligned}
$$


Proof. The proof is an easy calculation. We also note that for $f, g \in \mathcal{F}_{G}, \mathcal{E}(f, g)=\mathcal{E}^{c}(f, g)+$ $\int_{G \times G}(f(x)-f(y))(g(x)-g(y)) J(d x, d y)+\int_{G} f(x) g(x) \kappa^{G}(d x)$, where $\kappa^{G}=\kappa+\frac{1}{2} N(x, E \backslash G) \mu_{H}$.

Proof of Theorem 3.1. As in the analogous argument in [10], we can construct a common $\mathcal{E}$ nest $\left\{F_{n}\right\}_{n \in \mathbb{N}}$ (of compact sets) such that (i) $1_{F_{n}}\left(\mu_{\langle M\rangle}+\mu_{\langle\widehat{M}\rangle}+|\mu|\right) \in \mathbf{K}_{0}(X)$ for each $n \in \mathbb{N}$ and (ii) there exists $\rho_{n} \in \mathcal{F}$ such that $\Lambda\left(\widehat{M}^{c}\right)_{t}=N_{t}^{\rho_{n}}-\int_{0}^{t} \rho_{n}\left(X_{s}\right) d s$ for $0 \leq t<\tau_{F_{n}} \mathbf{P}_{m^{-}}$-a.s., $\left.\rho_{n}\right|_{F_{n}} \in C\left(F_{n}\right)$ and $1_{F_{n}} \mu_{\left\langle\rho_{n}\right\rangle} \in \mathbf{K}_{0}(X)$ for each $n \in \mathbb{N}$. Here we use the fact $\Lambda\left(1_{F_{n}} * \widehat{M}^{c}\right)_{t}=\Lambda\left(\widehat{M}^{c}\right)_{t}$ for $0 \leq t<\tau_{F_{n}}$ $\mathbf{P}_{m}$-a.s. and $\mu_{\left\langle\widehat{M}^{c}\right\rangle}\left(F_{n}\right)<\infty$. The latter implies that $1_{F_{n}} * \widehat{M}^{c}$ is an MAF of finite energy under $X$ and there exists a $\rho_{n} \in \mathcal{F}$ such that $\Lambda\left(1_{F_{n}} * \widehat{M}^{c}\right)_{t}=\Gamma\left(1_{F_{n}} * \widehat{M}^{c}\right)_{t}=N_{t}^{\rho_{n}}-\int_{0}^{t} \rho_{n}\left(X_{s}\right) d s$.

Let $E_{n}$ denote the fine interior of $F_{n}$ and define $\mathcal{F}^{(n)}:=\left\{u \in \mathcal{F} \mid u=0 \quad \mathcal{E}\right.$-q.e. on $\left.E_{n}^{c}\right\}$, and let $\mathcal{Q}^{(n)}$ denote the restriction of $\mathcal{Q}$ to $\mathcal{F}^{(n)}$. Clearly $\left(\mathcal{Q}^{(n)}, \mathcal{F}^{(n)}\right)$ is a quasi-regular positivity preserving coercive closed form on $L^{2}\left(E_{n} ; m\right)$ satisfying the same hypothesis as $(\mathcal{Q}, \mathcal{F})$. In fact, $\left(\mathcal{Q}^{(n)}, \mathcal{F}^{(n)}\right)$ is related to the restriction of $\mathcal{E}$ to $\mathcal{F}^{(n)}$ (which is the Dirichlet form of the part process $X^{E_{n}}$ ) in exactly the same way that $(\mathcal{Q}, \mathcal{F})$ is related to $(\mathcal{E}, \mathcal{F})$.

(i) First assume that $|\varphi|$ and $|\psi|$ are bounded above and below away from 0 . Note that $1_{F_{n}} N(|\varphi|+|\psi|) \mu_{H} \in \mathbf{K}_{0}(X)$ because of the boundedness of $|\varphi|$ and $|\psi|$ away from 0 . Then the conditions of Lemmas 3.2 and 3.3 are satisfied by $X^{E_{n}}$ and $\left(\mathcal{Q}^{(n)}, \mathcal{F}^{(n)}\right)$. Let $\left(Q_{t}^{(n)}\right)_{t>0}$ and $\left(V_{\alpha}^{(n)}\right)_{\alpha>\alpha_{0}}$ be the semigroup and resolvent on $L^{2}\left(E_{n} ; m\right)$ associated with $\left(\mathcal{Q}^{(n)}, \mathcal{F}^{(n)}\right)$. Let $\left(L^{\mathcal{Q},(n)}, \mathcal{D}\left(L^{\mathcal{Q},(n)}\right)\right)$ denote the infinitesimal generator of $\left(Q_{t}^{(n)}\right)$.

Consider a bounded $f \in \mathcal{D}\left(L^{\mathcal{Q},(n)}\right)$. Let $\tau_{n}$ be the first exit time of $X$ from $E_{n}$. Then by Lemma 3.3 (applied to $X^{E_{n}}$ and $\left(\mathcal{Q}^{(n)}, \mathcal{F}^{(n)}\right)$ ),

$$
\begin{aligned}
f\left(X_{t \wedge \tau_{n}}\right) Z_{t \wedge \tau_{n}}=f\left(X_{0}\right) & +\int_{0}^{t \wedge \tau_{n}} Z_{s-} d\left(M_{s}^{f,(n)}+U_{s}^{f,(n)}\right) \\
& +\int_{0}^{t \wedge \tau_{n}} Z_{s-} f\left(X_{s-}\right) d\left(M_{s}^{c,(n)}-\widehat{M}_{s}^{c,(n)}+W_{s}^{(n)}\right)+\int_{0}^{t \wedge \tau_{n}} Z_{s} L^{\mathcal{Q},(n)} f\left(X_{s}\right) d s,
\end{aligned}
$$

because $f\left(X_{\tau_{n}}\right)=0, \mathbf{P}_{m}$-a.s. Here was used the fact that $Z^{(n)}\left(\right.$ resp. $\left.M^{c,(n)}, W^{(n)}, U^{f,(n)}\right)$, the analog of $Z$ (resp. $M^{c}, W, U^{f}$ ) with respect to $X^{E_{n}}$ and $\left(\mathcal{Q}^{(n)}, \mathcal{F}^{(n)}\right)$, coincides with $Z$ (resp. $M^{c}$, $\left.W, U^{f}\right)$ on $\left[0, \tau_{n}\left[\right.\right.$. Let $\left\{T_{k}\right\}$ be an increasing sequence of $\left(\mathcal{F}_{t}\right)$-stopping times with $T_{k} \uparrow \zeta$ as $k \rightarrow \infty$ reducing the local martingale terms on the right hand side of (3.19). Replacing $t$ by $t \wedge T_{k}$ in (4.2) and taking expectations we obtain

$$
\mathbf{E}_{x}\left[f\left(X_{t \wedge T_{k} \wedge \tau_{n}}\right) Z_{t \wedge T_{k} \wedge \tau_{n}}\right]=f(x)+\mathbf{E}_{x}\left[\int_{0}^{t \wedge T_{k} \wedge \tau_{n}} Z_{s}\left(L^{\mathcal{Q},(n)} f\right)\left(X_{s}\right) d s\right],
$$

for $m$-a.e. $x \in E_{n}$. Hence, for non-negative $g \in L^{2}\left(E_{n} ; m\right)$ we have

$$
\mathbf{E}_{g m}\left[f\left(X_{t \wedge T_{k} \wedge \tau_{n}}\right) Z_{t \wedge T_{k} \wedge \tau_{n}}\right]=(f, g)_{L^{2}\left(E_{n} ; m\right)}+\mathbf{E}_{g m}\left[\int_{0}^{t \wedge T_{k} \wedge \tau_{n}} Z_{s}\left(L^{\mathcal{Q},(n)} f\right)\left(X_{s}\right) d s\right]
$$

Because $f$ is bounded, Lemma 3.2(ii) for $1_{E_{n}}, g \in L^{2}\left(E_{n} ; m\right)$ permits us to conclude that, as $k \rightarrow \infty$, 
the left side of (4.4) converges to $\mathbf{E}_{g m}\left[f\left(X_{t \wedge \tau_{n}}\right) Z_{t \wedge \tau_{n}}\right]$. On the other hand, because

$$
\left|\mathbf{E}_{x}\left[\int_{0}^{t \wedge \tau_{n}} Z_{s}\left(L^{\mathcal{Q},(n)} f\right)\left(X_{s}\right) d s\right]\right| \leq \int_{0}^{t} T_{s}^{(n)}\left|L^{\mathcal{Q},(n)} f\right|(x) d s,
$$

the left hand side of $(4.5)$ is in $L^{2}\left(E_{n} ; m\right)$ from Lemma 3.2(i), where

$$
T_{s}^{(n)} f(x):=\mathbf{E}_{x}\left[f\left(X_{s}\right) Z_{s}: s<\tau_{n}\right]
$$

Passing to the limit in (4.4) as $k \rightarrow \infty$, we obtain

$$
\mathbf{E}_{g m}\left[f\left(X_{t \wedge \tau_{n}}\right) Z_{t \wedge \tau_{n}}\right]=(f, g)_{L^{2}\left(E_{n} ; m\right)}+\mathbf{E}_{g m}\left[\int_{0}^{t \wedge \tau_{n}} Z_{s}\left(L^{\mathcal{Q},(n)} f\right)\left(X_{s}\right) d s\right]
$$

first for non-negative, and then for all $g \in L^{2}\left(E_{n} ; m\right)$. Then

$$
\mathbf{E}_{x}\left[f\left(X_{t \wedge \tau_{n}}\right) Z_{t \wedge \tau_{n}}\right]=f(x)+\mathbf{E}_{x}\left[\int_{0}^{t \wedge \tau_{n}} Z_{s}\left(L^{\mathcal{Q},(n)} f\right)\left(X_{s}\right) d s\right],
$$

for $m$-a.e. $x \in E_{n}$, provided $f \in \mathcal{D}\left(L^{\mathcal{Q},(n)}\right)$ is bounded. For $f \in \mathcal{D}\left(L^{\mathcal{Q},(n)}\right)$ of the form $V_{\alpha}^{(n)} g$ $\left(0 \leq g \in L^{2}\left(E_{n} ; m\right)\right)$, by the construction of the nest $\left\{F_{n}\right\}$, there is a sequence $\left\{g_{k}\right\}$ of non-negative elements of $L^{2}(E ; m)$ such that $f_{k}:=V_{\alpha}^{(n)} g_{k}$ is in $L^{\infty}\left(E_{n} ; m\right), f_{k}$ converges in $L^{2}\left(E_{n} ; m\right)$ to $f$ and $L^{\mathcal{Q},(n)} f_{k}=\alpha f_{k}-g_{k}$ converges to $L^{\mathcal{Q},(n)} f$ in $L^{2}\left(E_{n} ; m\right)$. [The boundedness of $f_{k}$ comes from the inequality $V_{\alpha}^{(n)} f \leq 1_{F_{n}} \cdot V_{\alpha} f$ for all non-negative $f \in L^{2}(E ; m)$, which is a consequence of the fact that these resolvents are associated with quasi-regular positivity preserving forms (see the argument in [10]). Substituting $f_{k}$ for $f$ in (4.8) and then passing to the limit as $k \rightarrow \infty$, we see that (4.8) is valid for all $f \in \mathcal{D}\left(L^{\mathcal{Q},(n)}\right)$, since any such $f$ can be written as $V_{\alpha}^{(n)} g_{1}-V_{\alpha}^{(n)} g_{2}$ for non-negative $g_{1}, g_{2} \in L^{2}\left(E_{n} ; m\right)$. That is, we have,

$$
T_{t}^{(n)} f(x)=f(x)+\int_{0}^{t} T_{s}^{(n)}\left(L^{\mathcal{Q},(n)} f\right)(x) d s, \quad m \text {-a.e. } x \in E_{n},
$$

for all $f \in \mathcal{D}\left(L^{\mathcal{Q},(n)}\right)$. This implies the strong continuity of $T_{t}^{(n)}$ on $\mathcal{D}\left(L^{\mathcal{Q},(n)}\right)$, hence on $L^{2}\left(E_{n} ; m\right)$. Note that $T_{t}^{(n)}$ maps $L^{2}\left(E_{n} ; m\right)$ into itself by Lemma 3.2(i), because

$$
1_{E_{n}}\left(\mu_{\langle M\rangle}+\mu_{\langle\widehat{M}\rangle}+N(|\varphi|) \mu_{H}+N(|\psi|) \mu_{H}+|\mu|\right)
$$

is a Kato class smooth measure with respect to $X^{E_{n}}$. Hence

$$
\lim _{t \downarrow 0} \frac{T_{t}^{(n)} f-f}{t}=L^{\mathcal{Q},(n)} f .
$$

Thus, using $\left(L^{(n)}, \mathcal{D}\left(L^{(n)}\right)\right)$ to denote the infinitesimal generator of $\left(T_{t}^{(n)}\right)_{t>0}$,

$$
\mathcal{D}\left(L^{\mathcal{Q},(n)}\right) \subset \mathcal{D}\left(L^{(n)}\right) \text { and } L^{\mathcal{Q},(n)}=L^{(n)} \text { on } \mathcal{D}\left(L^{\mathcal{Q},(n)}\right) .
$$


Let $G_{\alpha}^{(n)}:=\int_{0}^{\infty} e^{-\alpha t} T_{t}^{(n)} d t$ be the resolvent operator associated with $L^{(n)}$. Fix $u \in L^{2}\left(E_{n} ; m\right)$ and define, for $\alpha>\alpha_{0}, v:=V_{\alpha}^{(n)} u$. Then $v \in \mathcal{D}\left(L^{\mathcal{Q},(n)}\right) \subset \mathcal{D}\left(L^{(n)}\right)$ and $L^{(n)} v=L^{\mathcal{Q},(n)} v=\alpha v-u$. But $\mathcal{D}\left(L^{(n)}\right)$ coincides with $G_{\alpha}^{(n)}\left(L^{2}\left(E_{n} ; m\right)\right)$, so there exists $g \in L^{2}\left(E_{n} ; m\right)$ such that $v=G_{\alpha}^{(n)} g$, in which case $L^{(n)} v=\alpha v-g$. It follows that $u=g$, and then that $G_{\alpha}^{(n)} u=V_{\alpha}^{(n)} u$, for all $u \in L^{2}\left(E_{n} ; m\right)$. This identity of resolvents implies that the associated semigroups $\left(T_{t}^{(n)}\right)$ and $\left(Q_{t}^{(n)}\right)$ coincide under the boundedness of $|\varphi|,|\psi|$ away from 0 .

(ii) Secondly, we assume only that $\psi$ is bounded below away from 0 . For general $\varphi>-1$, define

$$
\varphi_{\ell}(x, y):=\left(1_{\left\{\frac{1}{\ell}<|\varphi|<\ell\right\}} \varphi\right)(x, y) .
$$

Clearly $\varphi_{\ell}$ satisfies the condition for $\varphi$ in step (i). Let $M^{d, \ell}$ be a purely discontinuous MAF on $I(\zeta)$ such that $\left.\Delta M_{t}^{d, \ell}=\varphi_{\ell}\left(X_{t-}, X_{t}\right), t \in\right] 0, \zeta\left[\right.$, and set $M^{\ell}:=M^{c}+M^{d, \ell}$. Then we see $\mu_{\left\langle M^{\ell}-M\right\rangle}=N\left(\left(\varphi_{\ell}-\varphi\right)^{2}\right) \mu_{H} \leq N\left(\varphi^{2}\right) \mu_{H}$. Hence $M^{\ell}$ converges uniformly to $M$ on any compact subinterval of $\left[0, \tau_{F_{n}}\left[\mathbf{P}_{m^{-}}\right.\right.$-a.s., because of the convergence of energy $\mathbf{e}\left(1_{F_{n}} *\left(M^{\ell}-M\right)\right) \rightarrow 0$. By replacing $M$ with $M^{\ell}$, we consider $\mathcal{Q}^{\ell}, Q_{t}^{\ell}, Z_{t}^{\ell}, T_{t}^{\ell}, G_{\alpha}^{\ell}$ instead of $\mathcal{Q}, Q_{t}, Z_{t}, T_{t}, G_{\alpha}$, respectively and also consider $Q_{t}^{\ell,(n)}, T_{t}^{\ell,(n)}, G_{\alpha}^{\ell,(n)}$ instead of $Q_{t}^{(n)}, T_{t}^{(n)}, G_{\alpha}^{(n)}$ respectively. From (i), we already know that $Q_{t}^{\ell,(n)}$ coincides with $T_{t}^{\ell,(n)}$. To show the coincidence, we first prove that $T_{t}^{(n)} f \in L^{2}\left(E_{n} ; m\right)$ and $T_{t}^{\ell,(n)} f$ weakly converges to $T_{t}^{(n)} f$ for any Borel function $f \in L^{2}\left(E_{n} ; m\right)$.

In order to prove this weak convergence, we will follow the approach in Chen and Zhang [8] by showing that there exists a constant $\check{\alpha}_{0}$ independent of $\ell, \ell_{0}$ with $\ell \geq \ell_{0}$ such that for any nonnegative Borel $f, g \in L^{2}\left(E_{n} ; m\right)$

$$
\sup _{\ell \geq \ell_{0}} \mathbf{E}_{m}\left[f\left(X_{t}\right) g\left(X_{0}\right) Z_{t}^{\ell_{0}}\left(Y_{t}^{\ell}\right)^{2}: t<\tau_{n}\right] \leq e^{\check{\alpha}_{0} t}\|f\|_{2}\|g\|_{2},
$$

where $Y_{t}^{\ell}:=Z_{t}^{\ell}\left(Z_{t}^{\ell_{0}}\right)^{-1}=\operatorname{Exp}\left(M_{t}^{d, \ell}\right) \operatorname{Exp}\left(M_{t}^{d, \ell_{0}}\right)^{-1}=\operatorname{Exp}\left(M_{t}^{d, \ell}-M_{t}^{d, \ell_{0}}\right)$ for $\ell \geq \ell_{0} \geq 1$. Here we use $\left[M^{d, \ell_{0}}, M^{d, \ell}-M^{d, \ell_{0}}\right]_{t}=0$ for $\ell \geq \ell_{0} \geq 1$. From (4.12) we see the uniform integrability of $\left\{Y_{t}^{\ell}, \ell \geq \ell_{0}\right\}$ under the law $1_{\left\{t<\tau_{n}\right\}} f\left(X_{t}\right) g\left(X_{0}\right) Z_{t}^{\ell_{0}} \mathbf{P}_{m}$, which implies the desired weak convergence. Indeed, from (i), we can conclude $\left\|T_{t}^{\ell,(n)}\right\|_{2 \rightarrow 2} \leq e^{\alpha_{0}} t$, hence $\left\|G_{\alpha}^{\ell,(n)}\right\|_{2 \rightarrow 2} \leq 1 /\left(\alpha-\alpha_{0}\right)$. By Fatou's lemma we have for Borel $f \in L^{2}\left(E_{n} ; m\right)$

$$
\int_{E_{n}}\left|T_{t}^{(n)} f(x)\right|^{2} m(d x) \leq \varliminf_{l \rightarrow \infty} \int_{E_{n}}\left|T_{t}^{\ell,(n)} f(x)\right|^{2} m(d x) \leq e^{\alpha_{0} t}\|f\|_{2}^{2} .
$$

Hence $\left\|T_{t}^{(n)}\right\|_{2 \rightarrow 2} \leq e^{\alpha_{0}} t$ and $\left\|G_{\alpha}^{(n)}\right\|_{2 \rightarrow 2} \leq 1 /\left(\alpha-\alpha_{0}\right)$. By (4.12), for each $f \in L^{2}\left(E_{n} ; m\right), T_{t}^{\ell,(n)} f$ converges to $T_{t}^{(n)} f$ weakly on $L^{2}\left(E_{n} ; m\right)$ and consequently $G_{\alpha}^{\ell,(n)} f$ converges to $G_{\alpha}^{(n)} f$ weakly on $L^{2}\left(E_{n} ; m\right)$ as $\ell \rightarrow \infty$. 
We now prove (4.12). Since

$$
\begin{aligned}
\left(Y_{t}^{\ell}\right)^{2}= & \left(\operatorname{Exp}\left(M_{t}^{d, \ell}-M_{t}^{d, \ell_{0}}\right)\right)^{2} \\
= & \exp \left(2 M_{t}^{d, \ell}-2 M_{t}^{d, \ell_{0}}\right) \prod_{0<s \leq t}\left(1+\Delta\left(M^{d, \ell}-M^{d, \ell_{0}}\right)_{s}\right)^{2} \exp \left(-2 \Delta\left(M^{d, \ell}-M^{d, \ell_{0}}\right)_{s}\right) \\
= & \exp \left(2 M_{t}^{d, \ell}-2 M_{t}^{d, \ell_{0}}\right) \prod_{0<s \leq t}\left(1+\left(1+\Delta\left(M^{d, \ell}-M^{d, \ell_{0}}\right)_{s}\right)^{2}-1\right) \\
& \quad \times \exp \left(1-\left(1+\Delta\left(M^{d, \ell}-M^{d, \ell_{0}}\right)_{s}\right)^{2}\right) \exp \left(\left(\Delta\left(M^{d, \ell}-M^{d, \ell_{0}}\right)_{s}\right)^{2}\right) \\
= & \operatorname{Exp}\left(K_{t}^{\ell}\right) \exp \left(\left(N\left(1_{\left\{1 / \ell<|\varphi| \leq 1 / \ell_{0}, \ell_{0} \leq|\varphi|<\ell\right\}} \varphi^{2}\right) * H\right)_{t}\right)
\end{aligned}
$$

where $K_{t}^{\ell}$ is the purely discontinuous MAF on $I(\zeta)$ with

$$
\begin{aligned}
\Delta K_{s}^{\ell} & =\left(1+\Delta\left(M^{d, \ell}-M^{d, \ell_{0}}\right)_{s}\right)^{2}-1 \\
& =2 \Delta\left(M^{d, \ell}-M^{d, \ell_{0}}\right)_{s}+\left(\Delta\left(M^{d, \ell}-M^{d, \ell_{0}}\right)_{s}\right)^{2} \\
& \left.=1_{\left\{1 / \ell<|\varphi| \leq 1 / \ell_{0}, \ell_{0} \leq|\varphi|<\ell\right\}}\left(2 \varphi+\varphi^{2}\right)\left(X_{s-}, X_{s}\right), \quad s \in\right] 0, \zeta[.
\end{aligned}
$$

Thus $\check{Z}_{t}^{\ell}:=Z_{t}^{\ell} Y_{t}^{\ell}=Z_{t}^{\ell_{0}}\left(Y_{t}^{\ell}\right)^{2}$ is of the same form as $Z_{t}^{\ell}$. Indeed,

$$
\begin{aligned}
\check{Z}_{t}^{\ell}= & \operatorname{Exp}\left(\widehat{M}_{t}\right) \circ r_{t} \operatorname{Exp}\left(M_{t}^{\ell_{0}}+K_{t}^{\ell}+A_{t}^{\mu}+\left(N\left(1_{\left\{1 / \ell<|\varphi| \leq 1 / \ell_{0}\right\}} \varphi^{2}\right) * H\right)_{t}+\left\langle M^{c}, \widehat{M}^{c}\right\rangle_{t}\right) \\
& \times\left(1+\psi\left(X_{t}, X_{t-}\right)\right)
\end{aligned}
$$

and the corresponding form $\check{\mathcal{Q}}^{\ell}$ on $\mathcal{F}^{(n)}$ is given by

$$
\begin{aligned}
\check{\mathcal{Q}}^{\ell}(f, g)= & \mathcal{E}(f, g)-\int_{E_{n}} f(x) \mu_{\left\langle M^{g}, \widehat{M}\right\rangle}^{E_{n}}(d x)-\int_{E_{n}} g(x) \mu_{\left\langle M^{f}, M^{\ell_{0}}+K_{t}^{\ell}\right\rangle}^{E_{n}}(d x) \\
& -\int_{E_{n}} f(x) g(x)\left(\mu^{E_{n}}+N\left(1_{E_{n} \times E_{n}} 1_{\left\{1 / \ell<|\varphi| \leq 1 / \ell_{0}, \ell_{0} \leq|\varphi|<\ell\right\}} \varphi^{2}\right) \mu_{H}\right)(d x) \\
& -\int_{E_{n} \times E_{n}} f(y) g(x) 1_{\left\{1 / \ell<|\varphi| \leq 1 / \ell_{0}, \ell_{0} \leq|\varphi|<\ell\right\}} \varphi(x, y) \psi(y, x) N(x, d y) \mu_{H}(d x)
\end{aligned}
$$

for $f, g \in \mathcal{F}^{(n)}$. Then the constant $\check{\delta}_{0}^{\ell}$ corresponding to $\check{\mathcal{Q}}^{\ell}$ on $\mathcal{F}^{(n)}$ is given by

$$
\begin{aligned}
\check{\delta}_{0}^{\ell}:= & \sqrt{2 \delta\left(1_{E_{n}} \mu_{\left\langle M^{\ell_{0}}+K_{t}^{\ell}\right\rangle}\right)}+\sqrt{2 \delta\left(1_{E_{n}} \mu_{\langle\widehat{M}\rangle}\right)}+\delta\left(1_{E_{n}} \mu^{+}\right) \\
& +\delta\left(1_{\left\{1 / \ell<|\varphi| \leq 1 / \ell_{0}, \ell_{0} \leq|\varphi|<\ell\right\}} 1_{E_{n} \times E_{n}} \varphi^{2}\right)+\sqrt{\delta\left(1_{\left\{1 / \ell<|\varphi| \leq 1 / \ell_{0}, \ell_{0} \leq|\varphi|<\ell\right\}} 1_{E_{n} \times E_{n}} \varphi^{2}\right)} \sqrt{\delta\left(1_{E_{n} \times E_{n}} \psi^{2}\right)}
\end{aligned}
$$

and it is bounded from above by

$$
\begin{aligned}
\check{\delta}_{0}:= & \sqrt{2 \delta\left(10\left(1_{E_{n}} \mu_{\langle M\rangle}\right)\right)}+\sqrt{2 \delta\left(1_{E_{n}} \mu_{\langle\widehat{M}\rangle}\right)}+\delta\left(1_{E_{n}} \mu^{+}\right)+\delta\left(1_{E_{n} \times E_{n}} \varphi^{2}\right) \\
& +\sqrt{\delta\left(1_{E_{n} \times E_{n}} \varphi^{2}\right)} \sqrt{\delta\left(1_{E_{n} \times E_{n}} \psi^{2}\right)} .
\end{aligned}
$$

Here we use $\left[M^{\ell_{0}}, K^{\ell}\right]=0,\left\langle M^{\ell_{0}}\right\rangle \leq\langle M\rangle$ and $\left\langle K^{\ell}\right\rangle \leq\langle 3 M\rangle$. Note that $\check{\delta}_{0}$ can be taken to be less than 1 because $1_{E_{n}}\left(\mu_{\langle M\rangle}+\mu_{\langle\widehat{M}\rangle}+|\mu|\right) \in \mathbf{K}(X)$. Therefore by (i) above and [13, Proposition 3.1], we have (4.12). 
Next we show that $\left\{G_{\alpha}^{(n)}, \alpha>\alpha_{0}\right\}$ is the resolvent associated with $\left(\mathcal{Q}^{(n)}, \mathcal{F}^{(n)}\right)$. Fix a bounded Borel $f \in L^{2}\left(E_{n} ; m\right)$. We easily see

$$
\mathcal{E}_{1}\left(G_{\alpha}^{\ell,(n)} f, G_{\alpha}^{\ell,(n)} f\right) \leq M \mathcal{Q}_{\alpha}^{\ell,(n)}\left(G_{\alpha}^{\ell,(n)} f, G_{\alpha}^{\ell,(n)} f\right)=M\left(f, G_{\alpha}^{\ell,(n)} f\right) \leq \frac{M}{\alpha-\alpha_{0}}\|f\|_{2}^{2} .
$$

So $\left\{G_{\alpha}^{\ell,(n)} f, n \in \mathbb{N}\right\}$ is $\mathcal{E}_{1}^{1 / 2}$-bounded. Taking a subsequence if necessary, $G_{\alpha}^{\ell,(n)} f$ converges weakly to some $f_{0} \in \mathcal{F}^{(n)}$ and its Cesàro mean strongly converges to $f_{0}$. Hence $f_{0}=G_{\alpha}^{(n)} f$. Since

$$
\begin{aligned}
& \mathcal{Q}^{\ell,(n)}(u, v)-\mathcal{Q}^{(n)}(u, v) \\
& \quad=-\int_{E_{n}} v d \mu_{\left\langle M^{u}, M^{\ell, d}-M^{d}\right\rangle}^{E_{n}}-\int_{E_{n} \times E_{n}} u(y) v(x)\left(\left(\varphi_{\ell}-\varphi\right) \cdot \bar{\psi}\right)(x, y) N(x, d y) \mu_{H}(d x),
\end{aligned}
$$

we have

$$
\begin{aligned}
& \left|\mathcal{Q}^{\ell,(n)}(u, v)-\mathcal{Q}^{(n)}(u, v)\right| \\
& \leq\left(\int_{E_{n}} v^{2} N\left(\varphi^{2}\right) d \mu_{H}\right)^{1 / 2}\left(\int_{E_{n} \times E_{n}}(u(x)-u(y))^{2} 1_{\{|\varphi| \leq 1 / \ell, \ell \leq|\varphi|\}}(x, y) N(x, d y) \mu_{H}(d x)\right)^{1 / 2} \\
& \quad+\left(\int_{E_{n}} v^{2} N\left(\varphi^{2}\right) d \mu_{H}\right)^{1 / 2}\left(\int_{E_{n}} u^{2} N\left(1_{\{|\bar{\varphi}| \leq 1 / \ell, \ell \leq|\bar{\varphi}|\}} \psi^{2}\right) d \mu_{H}\right)^{1 / 2}
\end{aligned}
$$

Taking $u:=G_{\alpha}^{(n)} f, v:=g_{\ell}:=G_{\alpha}^{\ell,(n)} f-G_{\alpha}^{(n)} f$ and noting $\sup _{\ell \in \mathbb{N}} \mathcal{E}_{1}\left(g_{\ell}, g_{\ell}\right)<\infty$, we have

$$
\begin{aligned}
\mathcal{E}_{1}\left(G_{\alpha}^{\ell,(n)} f-G_{\alpha}^{(n)} f, G_{\alpha}^{\ell,(n)} f-G_{\alpha}^{(n)} f\right) \\
\leq M \mathcal{Q}_{\alpha}^{\ell,(n)}\left(G_{\alpha}^{\ell,(n)} f-G_{\alpha}^{(n)} f, G_{\alpha}^{\ell,(n)} f-G_{\alpha}^{(n)} f\right) \\
=M\left(\mathcal{Q}_{\alpha}^{(n)}\left(G_{\alpha}^{(n)} f, g_{\ell}\right)-\mathcal{Q}_{\alpha}^{\ell,(n)}\left(G_{\alpha}^{(n)} f, g_{\ell}\right)\right) \\
\quad=M\left|\mathcal{Q}^{\ell,(n)}\left(G_{\alpha}^{(n)} f, g_{\ell}\right)-\mathcal{Q}^{(n)}\left(G_{\alpha}^{(n)} f, g_{\ell}\right)\right| \\
\rightarrow 0 \quad \text { as } \ell \rightarrow \infty .
\end{aligned}
$$

We also see

$$
\begin{aligned}
\mathcal{Q}_{\alpha}^{(n)}\left(G_{\alpha}^{(n)} f, v\right) & =\lim _{\ell \rightarrow \infty} \mathcal{Q}_{\alpha}^{(n)}\left(G_{\alpha}^{\ell,(n)} f, v\right) \\
& =\lim _{\ell \rightarrow \infty}\left(\mathcal{Q}_{\alpha}^{(n)}\left(G_{\alpha}^{\ell,(n)} f, v\right)-\mathcal{Q}_{\alpha}^{\ell,(n)}\left(G_{\alpha}^{\ell,(n)} f, v\right)\right)+(f, v)_{L^{2}\left(E_{n} ; m\right)} \\
& =(f, v)_{L^{2}\left(E_{n} ; m\right)} .
\end{aligned}
$$

Now $G_{\alpha}^{(n)} f=V_{\alpha}^{(n)} f$ holds for any Borel $f \in L^{2}\left(E_{n} ; m\right)$. Therefore, we have the desired result for general $\varphi>-1$ and $\psi$ bounded below away from 0 .

(iii) Finally, we show the coincidence for general $\varphi, \psi>-1$. By duality, it suffices to prove the coincidence $\hat{G}_{\alpha}^{(n)} f=\hat{V}_{\alpha}^{(n)} f$ for Borel $f \in L^{2}\left(E_{n} ; m\right)$, where

$$
\hat{G}_{\alpha}^{(n)} f=\int_{0}^{\infty} e^{-\alpha t} \hat{T}_{t} f d t \quad \text { and } \quad \hat{T}_{t}^{(n)} f(x):=\mathbf{E}_{x}\left[Z_{t} \circ r_{t} f\left(X_{t}\right): t<\tau_{n}\right] .
$$


Considering the approximation $\psi_{\ell}:=1_{\{|\psi|>1 / \ell\}} \psi$ for $\psi$, we can apply the result in (ii) and the proof is quite similar with (ii) [The boundedness of $\psi$ away from 0 in (ii) is only used for the application of (i)]. Therefore, we have the coincidence $\hat{G}_{\alpha}^{(n)} f=\hat{V}_{\alpha}^{(n)} f$, hence $G_{\alpha}^{(n)} f=V_{\alpha}^{(n)} f$ for any Borel $f \in L^{2}\left(E_{n} ; m\right)$.

Note that by [13, Proposition 3.1], condition (3.8) yields a uniform $L^{2}$-operator bound on $T_{t}^{(n)}$ : there is a constant $\alpha_{0} \geq 0$ such that

$$
\left\|T_{t}^{(n)}\right\|_{2 \rightarrow 2} \leq e^{\alpha_{0} t} \quad \text { for every } n \geq 1 \text { and } t>0 .
$$

As in [10], by using Lemma 3.6 in [14], we have that $G_{\alpha}$ coincides with $V_{\alpha}$, consequently $T_{t}$ coincides with $Q_{t}$ by use of the positivity preserving property of $(\mathcal{Q}, \mathcal{F})$ (see Lemma 4.1 ).

\section{Feynman-Kac type formula for $\Lambda(M)$}

In this section, we show that Theorem 3.1 yields an extension of the Feynman-Kac formula for zero energy CAF $N^{u}$ studied by Chen and Zhang in [8], where $u$ is a function in $\mathcal{F}$ having Kato class energy measure $\mu_{\left\langle M^{u}\right\rangle}$.

Let $M$ be a locally square-integrable MAF on $I(\zeta)$. Let $\varphi$ be the jumping function for $M$, that is, $\varphi$ vanishes on the diagonal of $E_{\partial} \times E_{\partial}$ and $\Delta M_{t}=\varphi\left(X_{t-}, X_{t}\right)$ for every $\left.t \in\right] 0, \zeta_{p}\left[, \mathbf{P}_{m^{-}}\right.$a.s. We assume that $\varphi$ is defined on $E_{\partial} \times E_{\partial}$ and $\varphi(x, y)+\varphi(y, x)=0$ for $x, y \in E_{\partial}$, which includes the case $M=M^{u}$ for $u \in \mathcal{F}_{e}$. Under this assumption, we have $\mathbf{P}_{m}$-a.s.

$$
\left.\Lambda(M)_{t}=-\frac{1}{2}\left(M_{t}+M_{t} \circ r_{t}+\varphi\left(X_{t}, X_{t-}\right)\right) \quad \text { for } t \in\right] 0, \zeta[.
$$

We further assume that $\mu_{\langle M\rangle}$, the energy measure of quadratic variation process $\langle M\rangle$, is of Hardy class, and define

$$
\delta_{0}:=\left[2 \delta\left(\mu_{\left\langle M^{c}\right\rangle}\right)\right]^{1 / 2}+\left[2 \delta\left(\mu_{\left\langle M^{j}\right\rangle}\right)\right]^{1 / 2}+\frac{1}{2}\left[\delta\left(\mu_{\left\langle M^{k}\right\rangle}\right)\right]^{1 / 2},
$$

where $M^{j}$ is the purely jump part and $M^{k}$ is the killing part of $M$, respectively. Actually, $M^{d}, M^{j}$ and $M^{k}$ can be expressed as follows:

$$
\begin{aligned}
M_{t}^{d} & :=\lim _{k \rightarrow \infty} \lim _{\varepsilon \rightarrow 0}\left(\sum_{s \in] 0, t]} 1_{G_{k}}\left(X_{s-}\right)\left(\varphi 1_{\{|\varphi|>\varepsilon\}}\right)\left(X_{s-}, X_{s}\right)-\left(1_{G_{k}} N\left(\varphi 1_{\{|\varphi|>\varepsilon\}}\right) * H\right)_{t}\right), \\
M_{t}^{j} & :=\lim _{k \rightarrow \infty} \lim _{\varepsilon \rightarrow 0}\left(\sum_{s \in] 0, t]} 1_{G_{k}}\left(X_{s-}\right)\left(\varphi 1_{\{|\varphi|>\varepsilon\}}\right)\left(X_{s-}, X_{s}\right) 1_{\{s<\zeta\}}-\left(1_{G_{k}} N\left(1_{E \times E} \varphi 1_{\{|\varphi|>\varepsilon\}}\right) * H\right)_{t}\right), \\
M_{t}^{k} & :=\lim _{k \rightarrow \infty} \lim _{\varepsilon \rightarrow 0}\left(1_{G_{k}}\left(X_{\zeta-}\right)\left(\varphi 1_{\{|\varphi|>\varepsilon\}}\right)\left(X_{\zeta-}, \partial\right) 1_{\left\{t \geq \zeta_{i}\right\}}-\int_{0}^{t} 1_{G_{k}}\left(X_{s}\right)\left(1_{\{|\varphi|>\varepsilon\}} \varphi\right)\left(X_{s}, \partial\right) N\left(X_{s},\{\partial\}\right) d H_{s}\right) .
\end{aligned}
$$


Note $M^{d}=M^{j}+M^{k}$. Here $\left\{G_{k}\right\}$ is a nest of finely open Borel sets such that $1_{G_{k}} * M \in \mathcal{M}$ (see Proposition 2.17 in [3]). We also note that

$$
\left\langle M^{j}\right\rangle_{t}=\int_{0}^{t}\left(\int_{E} \varphi\left(X_{s}, y\right)^{2} N\left(X_{s}, d y\right)\right) d H_{s}, \quad\left\langle M^{k}\right\rangle_{t}=\int_{0}^{t} \varphi\left(X_{s}, \partial\right)^{2} N\left(X_{s},\{\partial\}\right) d H_{s},
$$

and

$$
\mu_{\left\langle M^{j}\right\rangle}(d x)=N\left(1_{E \times E} \varphi^{2}\right) \mu_{H}(d x), \quad \mu_{\left\langle M^{k}\right\rangle}(d x)=\varphi(x, \partial)^{2} N(x,\{\partial\}) \mu_{H}(d x) .
$$

We consider the following quadratic form $\mathcal{Q}$ on $\mathcal{F}$ : For $f, g \in \mathcal{F}$,

$$
\begin{aligned}
& \mathcal{Q}(f, g):=\mathcal{E}(f, g)+ \frac{1}{2} \int_{E} g d \mu_{\left\langle M^{f, c}, M^{c}\right\rangle}+\frac{1}{2} \int_{E} f d \mu_{\left\langle M^{g, c}, M^{c}\right\rangle} \\
&+\frac{1}{2} \int_{E} g(x)\left(\int_{E}(f(y)-f(x)) \varphi(x, y) N(x, d y)\right) \mu_{H}(d x) \\
&+\frac{1}{2} \int_{E} f(x)\left(\int_{E}(g(y)-g(x)) \varphi(x, y) N(x, d y)\right) \mu_{H}(d x) \\
&-\int_{E} f(x) g(x) \varphi(x, \partial) N(x,\{\partial\}) \mu_{H}(d x)
\end{aligned}
$$

From (5.1), we have (3.4) and (3.5) with

$$
\alpha_{0}:=\gamma\left(\mu_{\left\langle M^{c}\right\rangle}\right)\left(2 / \delta\left(\mu_{\left\langle M^{c}\right\rangle}\right)\right)^{1 / 2}+\gamma\left(\mu_{\left\langle M^{j}\right\rangle}\right)\left(2 / \delta\left(\mu_{\left\langle M^{j}\right\rangle}\right)\right)^{1 / 2}+\frac{1}{2} \gamma\left(\mu_{\left\langle M^{k}\right\rangle}\right)\left(1 / \delta\left(\mu_{\left\langle M^{k}\right\rangle}\right)\right)^{1 / 2} .
$$

Note that

$$
\mathcal{Q}(f, g)=\mathcal{E}(f, g)+\frac{1}{2} \nu_{\left\langle M^{f g}, M\right\rangle}(E) \quad \text { for } f, g \in \mathcal{F}_{b}
$$

where the signed measure $\nu_{\langle M, N\rangle}$ for locally square-integrable MAFs $M, N$ on $\llbracket 0, \zeta \llbracket$ (more strongly, on $I(\zeta))$ is defined by $\nu_{\langle M, N\rangle}:=\mu_{\left\langle M^{c}, N^{c}\right\rangle}+\mu_{\left\langle M^{j}, N^{j}\right\rangle}+2 \mu_{\left\langle M^{k}, N^{k}\right\rangle}$. We have the following result extending Theorem 1.2 in [8]. As with Theorem 3.1, we note that if the constant $\delta_{0}$ defined in (5.1) is strictly less than 1 , then condition (5.5) below is satisfied.

Theorem 5.1 Suppose that $\mu_{\langle M\rangle} \in \mathbf{H}(X)$, and that there are constants $\alpha>0$ and $c>1$ such that

$$
c^{-1} \mathcal{E}_{1}(u, u) \leq \mathcal{Q}_{\alpha}(u, u) \leq c \mathcal{E}_{1}(u, u) \quad \text { for } u \in \mathcal{F}_{b} .
$$

Then $\bar{P}_{t} f(x):=\mathbf{E}_{x}\left[e^{\Lambda(M)_{t}} f\left(X_{t}\right)\right]$ is the symmetric semigroup associated with $(\mathcal{Q}, \mathcal{F})$.

Proof. Note that $M=M^{c}+M^{d}$, where $M^{c}$ and $M^{d}$ are the continuous and purely discontinuous martingale parts of $M$. Let $M^{d, 1}$ and $M^{d, 2}$ be the purely discontinuous locally square integrable MAFs of $X$ with

$$
M_{t}^{d, 1}-M_{t-}^{d, 1}=\mathbf{1}_{\left\{\left|\varphi\left(X_{t-}, X_{t}\right)\right| \leq 1\right\}} \varphi\left(X_{t-}, X_{t}\right) \quad \text { and } \quad M_{t}^{d, 2}-M_{t-}^{d, 2}=\mathbf{1}_{\left\{\left|\varphi\left(X_{t-}, X_{t}\right)\right|>1\right\}} \varphi\left(X_{t-}, X_{t}\right),
$$


respectively. Let $(N, H)$ be a Lévy system of $X$. By (2.6) in [3], for $t<\zeta$,

$$
\begin{aligned}
\Lambda\left(M^{d, 2}\right)_{t} & =-\frac{1}{2}\left(M_{t}^{d, 2}+M_{t}^{d, 2} \circ r_{t}+\varphi\left(X_{t}, X_{t-}\right) \mathbf{1}_{\left\{\left|\varphi\left(X_{t}, X_{t-}\right)\right|>1\right\}}\right) \\
& =\int_{0}^{t} \int_{E} \mathbf{1}_{\left\{\left|\varphi\left(X_{s}, y\right)\right|>1\right\}} \varphi\left(X_{s}, y\right) N\left(X_{s}, d y\right) d H_{s},
\end{aligned}
$$

where in the last equality we have used the fact that $\varphi(x, y)=-\varphi(y, x)$ for $x, y \in E$. Thus $\Lambda\left(M^{d, 2}\right)$ is CAF of $X$ with signed Revuz measure

$$
\nu(d x):=\left(\int_{E} \mathbf{1}_{\{|\varphi(x, y)|>1\}} \varphi(x, y) N(x, d y)\right) \mu_{H}(d x) .
$$

Evidently,

$$
|\nu|(d x) \leq\left(\int_{E} \varphi(x, y)^{2} N(x, d y)\right) \mu_{H}(d x) \leq \mu_{\langle M\rangle}(d x) .
$$

Put $M^{1}:=M^{c}+M^{d, 1}$; this is a locally square integrable MAF of $X$ with bounded jumps, and

$$
\Lambda(M)=\Lambda\left(M^{1}\right)+\Lambda\left(M^{d, 2}\right)=\Lambda\left(M^{u, 1}\right)+A^{\nu} .
$$

Thus, without loss of generality, the jump function $\varphi$ of $M$ can be taken to be bounded in magnitude by 1 .

Note that $\left|e^{x}-1\right| \leq|x| \frac{e^{1 / 2}-1}{1 / 2}$ for $|x| \leq 1 / 2$. Let $J_{t}$ be the purely discontinuous locally squareintegrable MAF on $I(\zeta)$ satisfying $\left.\Delta J_{t}=\exp \left[-\frac{1}{2} \varphi\left(X_{t-}, X_{t}\right)\right]-1, t \in\right] 0, \zeta\left[\right.$ and set $\bar{M}_{t}:=-\frac{1}{2} M_{t}^{c}+J_{t}$ for $t \in I(\zeta)$. We have $\Delta \bar{M}_{t}=\Delta J_{t}>-1$ for $\left.t \in\right] 0, \zeta[$, and on $\{t<\zeta\}$

$$
e^{-\frac{1}{2} M_{t}}=\operatorname{Exp}\left(\bar{M}_{t}\right) e^{-A_{t}^{\mu}}
$$

where

$$
\mu(d x):=\int_{E_{\partial}} \mathbf{1}_{\{|\varphi| \leq 1\}}(x, y)\left(1-\frac{\varphi(x, y)}{2}-e^{-\frac{\varphi(x, y)}{2}}\right) N(x, d y) \mu_{H}(d x)-\frac{1}{8} \mu_{\left\langle M^{c}\right\rangle}(d x) .
$$

We also have, on $\{t<\zeta\}$,

$$
e^{\Lambda(M)_{t}}=\operatorname{Exp}\left(\bar{M}_{t}\right) \circ r_{t} \operatorname{Exp}\left(\bar{M}_{t}\right) e^{A_{t}^{\nu}-2 A_{t}^{\mu}} e^{-\varphi\left(X_{t}, X_{t-}\right) / 2} .
$$

The theorem now follows from Theorem 3.1.

\section{Examples}

Example 6.1 Let $X=\left(X_{t}, \mathbf{P}_{x}\right)_{x \in \mathbb{R}^{d}}$ be the symmetric $\alpha$-stable process with $\left.\alpha \in\right] 0,2[$ and $(\mathcal{E}, \mathcal{F})$ the corresponding Dirichlet form on $L^{2}\left(\mathbb{R}^{d}\right)$, and $K$ a compact subset of $\mathbb{R}^{d}$. It is well-known that $X$ is transient and has Green function given by $G(x, y):=\mathcal{A}(d, \alpha)|x-y|^{-(d-\alpha)}$ provided $d>\alpha$, where

$$
\mathcal{A}(d, \beta):=\frac{|\beta| \Gamma\left(\frac{d-\beta}{2}\right)}{2^{1+\beta} \pi^{d / 2} \Gamma\left(1+\frac{\beta}{2}\right)}, \quad \beta<d
$$


and $(\mathcal{E}, \mathcal{F})$ is given by

$$
\begin{aligned}
\mathcal{E}(u, v) & =\frac{\mathcal{A}(d,-\alpha)}{2} \int_{\mathbb{R}^{d} \times \mathbb{R}^{d}} \frac{(u(x)-u(y))(v(x)-v(y))}{|x-y|^{d+\alpha}} d x d y, \\
\mathcal{F} & =\left\{u \in L^{2}\left(\mathbb{R}^{d}\right) \mid \int_{\mathbb{R}^{d} \times \mathbb{R}^{d}} \frac{(u(x)-u(y))^{2}}{|x-y|^{d+\alpha}} d x d y<\infty\right\} .
\end{aligned}
$$

The process $X$ has a Lévy system $(N, H)$, where $N(x, d y):=\mathcal{A}(d,-\alpha)|x-y|^{-(d+\alpha)} d y$ and $H_{t}=t$. So $\mu_{H}(d x)=d x$.

We say that a measurable function $f$ on $\mathbb{R}^{d}$ is of Kato class (resp. Hardy class) if the measure $|f(x)| d x$ is of Kato class (resp. Hardy class) and write $f \in \mathbf{K}(X)$ (resp. $f \in \mathbf{H}(X)$ ). Since there exists $C>0$ such that $p_{t}(x, y) \leq C t^{-d / \alpha}$ for all $x, y \in \mathbb{R}^{d}$, we have $L^{p}\left(\mathbb{R}^{d}\right) \subset \mathbf{K}(X) \subset \mathbf{H}(X)$ if $p>d / \alpha($ resp. $p \geq 1)$ for the case $d>\alpha$ (resp. for $d=\alpha=1$ ) (see [15]). For $d>\alpha$, we have the following Sobolev inequality

$$
\|u\|_{\frac{2 d}{d-\alpha}} \leq C_{d, \alpha} \mathcal{E}(u, u)^{1 / 2} \quad \text { for } u \in \mathcal{F} .
$$

For every $f \in L^{d / \alpha}\left(\mathbb{R}^{d}\right)$ and every $\delta>0$, there is $\gamma>0$ such that $\left\|1_{\{|f|>\gamma\}} f\right\|_{d / \alpha} \leq \delta / C_{d, \alpha}^{2}$. So when $d>\alpha$, we have for $u \in \mathcal{F}$,

$$
\int_{\mathbb{R}^{d}} u(x)^{2}|f(x)| d x \leq\left\|u^{2}\right\|_{\frac{d}{d-\alpha}}\left\|1_{\{|f|>\gamma\}} f\right\|_{\frac{d}{\alpha}}+\gamma \int_{\mathbb{R}^{d}} u(x)^{2} d x \leq \delta \mathcal{E}(u, u)+\gamma \int_{\mathbb{R}^{d}} u(x)^{2} d x .
$$

This proves that, when $d>\alpha, L^{d / \alpha}\left(\mathbb{R}^{d}\right) \subset \mathbf{H}(X)$ and the coefficient $\delta(|f|)$ for $f \in L^{d / \alpha}\left(\mathbb{R}^{d}\right)$ can be taken to be arbitrarily small.

We assume one of the following:

- $\varphi_{i}>-1$ is a Borel function on $\mathbb{R}^{d} \times \mathbb{R}^{d}$ such that $\left|\varphi_{i}(x, y)\right| \leq C_{i} 1_{K}(x) 1_{K}(y)|x-y|^{\gamma_{i}}$ with $\gamma_{i}>\alpha / 2$ and $C_{i}>0, i=1,2$. Then $N\left(\varphi_{i}^{2}\right)$ is bounded, hence in $\mathbf{K}(X)$.

- $f_{i} \in L_{+}^{p}\left(\mathbb{R}^{d}\right)$ with $p \geq d / \alpha$ for $d>\alpha$, or with $p \geq 1$ for $d=\alpha=1$, and $\gamma_{i}>\alpha / 2, i=1,2$. We let $\varphi_{i}(x, y):=f_{i}(x)^{1 / 2} 1_{K}(x) 1_{K}(y)|x-y|^{\gamma_{i}}\left(\operatorname{resp} . \varphi_{i}(x, y):=f_{i}(x)^{1 / 2} 1_{K}(y)|x-y|^{\gamma_{i}}\right)$ when $p>d / \alpha($ resp. $p=d / \alpha), i=1,2$. Then $N\left(\varphi_{i}^{2}\right) \in L^{d / \alpha}\left(\mathbb{R}^{d}\right) \subset \mathbf{H}(X)$.

Let $M, \widehat{M}$ be locally square-integrable MAFs with $\Delta M_{t}=\varphi_{1}\left(X_{t-}, X_{t}\right)$ and $\Delta \widehat{M}_{t}=\varphi_{2}\left(X_{t-}, X_{t}\right)$ for $t>0$. Then by Theorem 3.1, $T_{t} f(x):=\mathbf{E}_{x}\left[Z_{t} f\left(X_{t}\right)\right]$ with $Z_{t}:=\operatorname{Exp}\left(\widehat{M}_{t}\right) \circ r_{t} \operatorname{Exp}\left(M_{t}\right)(1+$ $\left.\varphi_{2}\left(X_{t}, X_{t-}\right)\right)$ gives the strongly continuous semigroup associated with

$$
\begin{aligned}
\mathcal{Q}(f, g):= & \mathcal{E}(f, g)-\mathcal{A}(d,-\alpha) \int_{\mathbb{R}^{d}} g(x)\left(\int_{\mathbb{R}^{d}}(f(y)-f(x)) \varphi_{1}(x, y) \frac{d y}{|x-y|^{d+\alpha}}\right) d x \\
& -\mathcal{A}(d,-\alpha) \int_{\mathbb{R}^{d}} f(x)\left(\int_{\mathbb{R}^{d}}(g(y)-g(x)) \varphi_{2}(x, y) \frac{d y}{|x-y|^{d+\alpha}}\right) d x \\
& -\mathcal{A}(d,-\alpha) \int_{\mathbb{R}^{d} \times \mathbb{R}^{d}} f(y) g(x) \varphi_{1}(x, y) \varphi_{2}(y, x) \frac{d x d y}{|x-y|^{d+\alpha}} .
\end{aligned}
$$


Example 6.2 Let $X=\left(X_{t}, \mathbf{P}_{x}\right)_{x \in D}$ be the symmetric censored $\alpha$-stable process on a bounded Lipschitz domain $D$ with $d \geq 2>\alpha>1$ and $(\mathcal{E}, \mathcal{F})$ the corresponding Dirichlet form on $L^{2}(D)$. $(\mathcal{E}, \mathcal{F})$ on $L^{2}(D)$ is given by

$$
\begin{aligned}
\mathcal{E}(u, v) & =\frac{\mathcal{A}(d,-\alpha)}{2} \int_{D \times D} \frac{(u(x)-u(y))(v(x)-v(y))}{|x-y|^{d+\alpha}} d x d y, \\
\mathcal{F} & =W_{0}^{\alpha / 2,2}(D)
\end{aligned}
$$

where $W_{0}^{\alpha / 2,2}(D)$ is the completion of $C_{0}^{\infty}(D)$ with respect to the $\mathcal{E}_{1}^{1 / 2}$-norm and $\mathcal{A}(d,-\alpha)$ is the constant appeared in the previous example. $X$ has Lévy system $(N, H)$, where $H_{t}=t, N(x, d y):=$ $\mathcal{A}(d,-\alpha)|x-y|^{-(d+\alpha)} d y$ on $D$ and $N(x,\{\partial\})=0, x \in D$.

By Chen-Song [7], the following Hardy inequality holds:

$$
\int_{D} \frac{u(x)^{2}}{d(x, \partial D)^{\alpha}} d x \leq C_{D, \alpha} \mathcal{E}(u, u), \quad u \in \mathcal{F}
$$

Let $\varepsilon>2(2+\sqrt{6}) C_{D, \alpha}$ and for each $i=1,2$ and $\gamma_{i}>\alpha$, set

$$
\varphi_{i}(x, y):=\left(\sup _{x \in D} \int_{D-x}|z|^{\gamma_{i}-\alpha-1} d z\right)^{-1 / 2}\left(\frac{1}{\mathcal{A}(d,-\alpha)} \cdot \frac{1}{\left(C_{D, \alpha}+\varepsilon\right)} \frac{|x-y|^{\gamma_{i}}}{d(x, \partial D)^{\alpha}}\right)^{1 / 2} .
$$

Then $N\left(\varphi_{i}^{2}\right)(x)=\left(C_{D, \alpha}+\varepsilon\right)^{-1} d(x, \partial D)^{-\alpha}$ and so $\delta\left(\varphi_{i}^{2}\right) \leq C_{D, \alpha} /\left(C_{D, \alpha}+\varepsilon\right)$. Thus we have

$$
\delta_{0}:=\sqrt{2 \delta\left(\varphi_{1}^{2}\right)}+\sqrt{2 \delta\left(\varphi_{2}^{2}\right)}+\sqrt{\delta\left(\varphi_{1}^{2}\right) \delta\left(\varphi_{2}^{2}\right)}<1 .
$$

Let $M, \widehat{M}$ be locally square-integrable MAFs with $\Delta M_{t}=\varphi_{1}\left(X_{t-}, X_{t}\right)$ and $\Delta \widehat{M}_{t}=\varphi_{2}\left(X_{t-}, X_{t}\right)$ for $t \in] 0, \zeta\left[\right.$. Then by Theorem $3.1, T_{t} f(x):=\mathbf{E}_{x}\left[Z_{t} f\left(X_{t}\right)\right]$ with $Z_{t}:=\operatorname{Exp}\left(\widehat{M}_{t}\right) \circ r_{t} \operatorname{Exp}\left(M_{t}\right)(1+$ $\left.\varphi_{2}\left(X_{t}, X_{t-}\right)\right)$ gives the strongly continuous semigroup associated with

$$
\begin{aligned}
\mathcal{Q}(f, g):= & \mathcal{E}(f, g)-\mathcal{A}(d,-\alpha) \int_{D} g(x)\left(\int_{D}(f(y)-f(x)) \varphi_{1}(x, y) \frac{d y}{|x-y|^{d+\alpha}}\right) d x \\
& -\mathcal{A}(d,-\alpha) \int_{D} f(x)\left(\int_{D}(g(y)-g(x)) \varphi_{2}(x, y) \frac{d y}{|x-y|^{d+\alpha}}\right) d x \\
& -\mathcal{A}(d,-\alpha) \int_{D \times D} f(y) g(x) \varphi_{1}(x, y) \varphi_{2}(y, x) \frac{d x d y}{|x-y|^{d+\alpha}} .
\end{aligned}
$$

\section{References}

[1] Z.-Q. Chen: Analytic characterization of conditional gaugeability for non-local Feynman-Kac transform, J. Funct. Anal. 202 (2003), 226-246.

[2] Z.-Q. Chen, P. J. Fitzsimmons, M. Takeda, J. Ying and T.-S. Zhang: Absolute continuity of symmetric Markov processes, Ann. Probab. 32 (2004), 2067-2098.

[3] Z.-Q. Chen, P. J. Fitzsimmons, K. Kuwae and T.-S. Zhang: Stochastic calculus for Dirichlet processes, preprint, 2006. 
[4] Z.-Q. Chen, Z.-M. Ma and M. Röckner: Quasi-homeomorphisms of Dirichlet forms, Nagoya Math. J. 136 (1994), 1-15.

[5] Z.-Q. Chen and R. Song: Conditional gauge theorem for non-local Feynman-Kac transforms, Probab. Theory Relat. Fields 125 (2003), 45-72

[6] Z.-Q. Chen and R. Song: Drift transforms and Green function estimates for discontinuous processes, $J$. Funct. Anal. 201 (2003), 262-281.

[7] Z.-Q. Chen and R. Song: Hardy inequality for censored stable processes, Tohoku Math. J. 55 (2003), $439-450$.

[8] Z.-Q. Chen and T.-S. Zhang: Girsanov and Feynman-Kac type transformations for symmetric Markov processes, Ann. Inst. H. Poincaré Probab. Statist. 38 (2002), 475-505.

[9] P.J. Fitzsimmons: Absolute continuity of symmetric diffusions, Ann. Probab. 25 (1997), 230-258.

[10] P. J. Fitzsimmons and K. Kuwae: Non-symmetric perturbations of symmetric Dirichlet forms, J. Funct. Anal. 208 (2004) 140-162.

[11] M. Fukushima, Y. Ōshima and M. Takeda: Dirichlet Forms and Symmetric Markov Processes, de Gruyter, Berlin, 1994.

[12] S. W. He, J. G. Wang and J. A. Yan: Semimartingale Theory and Stochastic Calculus, Science Press, Beijing New York, 1992.

[13] H. Kunita, Sub-Markov semi-group in Banach lattices. Proc. International Conf. on Func. Analysis Relet. Topics. Tokyo (1969), 332-343.

[14] K. Kuwae: Maximum principles for subharmonic functions via local semi-Dirichlet forms, to appear in Canadian J. Math., 2006.

[15] K. Kuwae and M. Takahashi: Kato class functions of Markov processes under ultracontractivity, Potential Theory in Matsue, 193-202, Adv. Stud. Pure Math., 44, Math. Soc. Japan, Tokyo, 2006.

[16] J. Lunt, T. J. Lyons and T.-S. Zhang: Integrability of functionals of Dirichlet processes, probabilistic representations of semigroups, and estimates of heat kernels, J. Funct. Anal. 153 (1998), 320-342.

[17] Z.-M. Ma and M. Röckner: Introduction to the Theory of (Non-Symmetric) Dirichlet Forms, SpringerVerlag, Berlin, 1992.

[18] Z.-M. Ma and M. Röckner: Markov processes associated with positivity preserving coercive forms, Canad. J. Math. 47 (1995), 817-840.

[19] S. Nakao: Stochastic calculus for continuous additive functionals of zero energy, Z. Wahrsch. verw. Gebiete 68 (1985) 557-578.

[20] M. Sharpe: General Theory of Markov Processes, Academic Press, San Diego, 1988.

\section{Addresses:}

Zhen-Qing Chen: Department of Mathematics, University of Washington, Seattle, WA 98195, USA. Email: zchen@math.washington.edu

Patrick J. Fitzsimmons: Department of Mathematics, University of California at San Diego, La Jolla, CA 92093-0112, USA. Email: pfitzsim@ucsd.edu

Kazuhiro Kuwae: Department of Mathematics, Faculty of Education, Kumamoto University, Kumamoto 860-8555, Japan. Email: kuwae@gpo.kumamoto-u.ac.jp

Tusheng Zhang: School of Mathematics, University of Manchester, Sackville Street, Manchester M60 1QD, UK. Email: tzhang@maths.manchester.ac.uk 